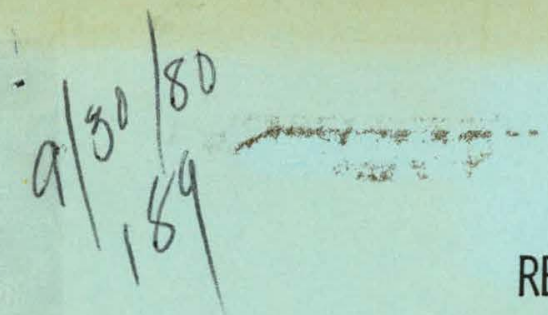

\title{
Dr. 1864
}

UC-2OF

RENSSELAER POLYTECHNIC INSTITUTE

Plasma Dynamics Laboratory

Troy, New YoRK 12181

"HEAVY ION BEAM PROBING"

MASTER

BY

R. L. HICKOK

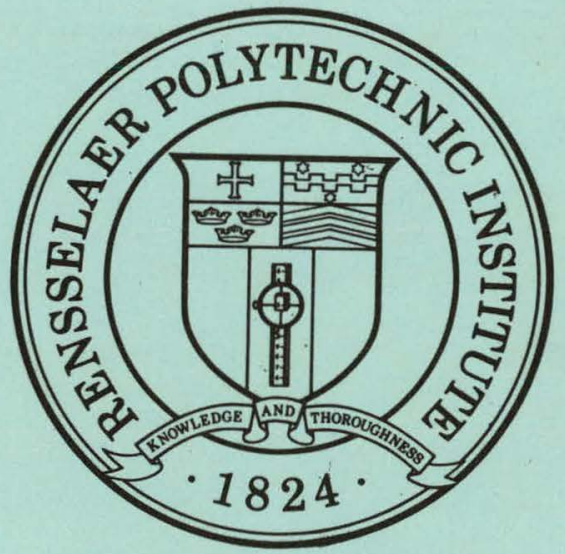

REPORT No: DOE/ET/53004-2

RPDL REPORT No, 80-14

JULY 1980 


\section{DISCLAIMER}

This report was prepared as an account of work sponsored by an agency of the United States Government. Neither the United States Government nor any agency Thereof, nor any of their employees, makes any warranty, express or implied, or assumes any legal liability or responsibility for the accuracy, completeness, or usefulness of any information, apparatus, product, or process disclosed, or represents that its use would not infringe privately owned rights. Reference herein to any specific commercial product, process, or service by trade name, trademark, manufacturer, or otherwise does not necessarily constitute or imply its endorsement, recommendation, or favoring by the United States Government or any agency thereof. The views and opinions of authors expressed herein do not necessarily state or reflect those of the United States Government or any agency thereof. 


\section{DISCLAIMER}

Portions of this document may be illegible in electronic image products. Images are produced from the best available original document. 


\section{DISCLAIMER}

This report was prepared as an account of work sponsored by an agency of the United States Government, Neither the United States Government nor any agency thereof, nor any of their employees, makes any warranty, express or implied, or assumes any legal liability or responsibility for the accuracy, completeness, or usefulness of any information, apparatus, product, or process disclosed, or represents that its use would not infringe privately owned rights. Reference herein to any specific commercial product, process, or service by trade name, trademark, manufacturer, or otherwise, does not necessarily constitute or imply its endorsement recommendation, or favoring by the United States Government or any agency thereof. The views and opinions of authors expressed herein do not necessarily state or reflect those of the United States Government or any agency thereof.

Printed in Lhe United States of America Available from

National Technical Information Service

U. S. Department of Commerce

5285 Port Royal Road

Springfield, VA 22161

NTIS price codes

Printed copy: $\$ 7.00$

Microfiche copy: A01 
Page

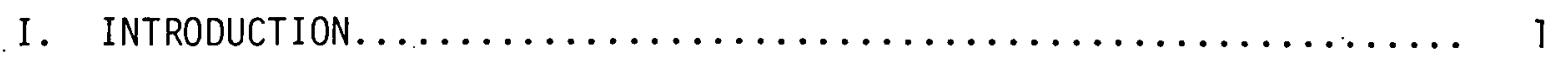

II. MAPPing the Plasma................................... 5

III. SPACE RESOLUTION................................. 8

IV. SPACE POTENTIAL MEASUREMENTS $\ldots \ldots \ldots \ldots \ldots \ldots \ldots \ldots \ldots \ldots \ldots \ldots \ldots \ldots \ldots$

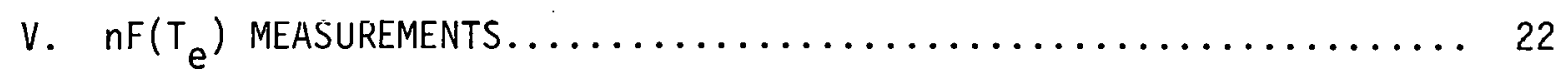

VI. TIME RESOLUTION.................................... 26

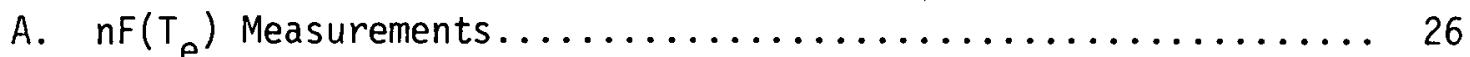

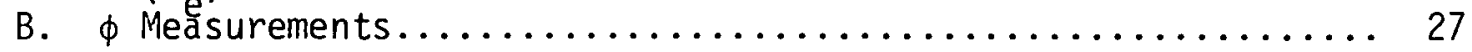

V.I . BETA AND MAGNETIC FIELD MEASUREMENTS $\ldots \ldots \ldots \ldots \ldots \ldots \ldots \ldots . \ldots \ldots$

VIII. PROBLEM AREAS.................................. 32

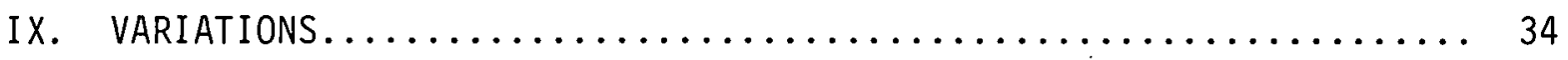

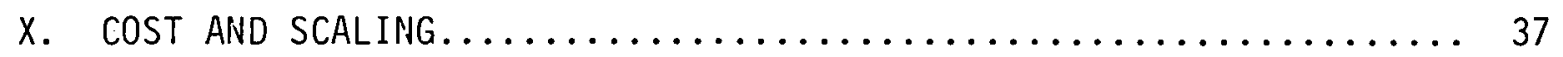

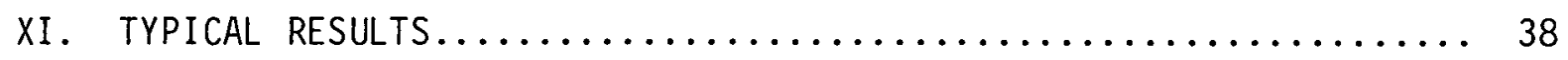

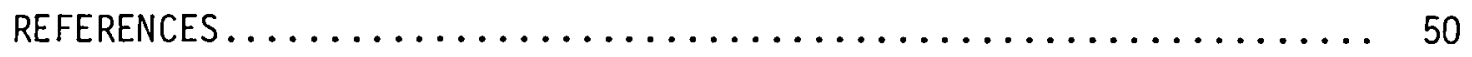

DISTRIBUTION LIST.............................. 52 


\section{ABSTRACT}

This report consists of the notes distributed to the participants at the IEEE Mini-Course on Modern Plasma Diagnostics that was held in Madison, Wisconsin in May 1980. It presents an overview of Heavy Ion Beam Probing that briefly describes the principles and discuss the types of measurements that can be made. The problems associated with implementing beam probes are noted, possible variations are described, estimated costs of present day systems, and the scaling requirements for large plasma devices are presented. The final chapter illustrates typical results that have been obtained on a variety of plasma devices. No detailed calculations are included in the report, but a list of references that will provide more detailed information is included. 


\section{INTRODUCTION}

Heavy ion beam probing is an active particle diagnostic system that can provide space and time resolved measurements of plasma density, electron temperature, and space potential. The measurements are continuous in time and quasi-continuous in space, and consequently is ideally suited for studying fluctuation phenomena. It is the only technique that can provide a direct measurement of the space potential in a high temperature magnetically confined plasma. Beam probing can also provide information on plasma beta and it may be able to provide information on the magnetic field structure, but this has not yet been demonstrated.

The physics of beam probing, which is illustrated in Fig. 1, is quite simple. A beam of singly charged ions, called the primary beam, is directed across the confining magnetic field and through the plasma. Some of the particles in the primary beam make ionizing collisions with the plasma electrons to form doubly charged secondary ions. There is essentially no momentum transfer in this collision due to the large mass difference between the probing ion and the plasma electron. The secondary ions will be created all along the primary beam path. Due to the increase in charge, the radius of curvature of the secondary ions will be half that of the primary beam. This will separate a secondary ion from the primary beam and from other secondary ions that are created at different points on the primary beam path. If we place a small aperature detector in the fan of secondary ions emerging from the plasma, it will only observe those secondaries that are created in a small sample volume centered at a known location in the plasma. In effect 


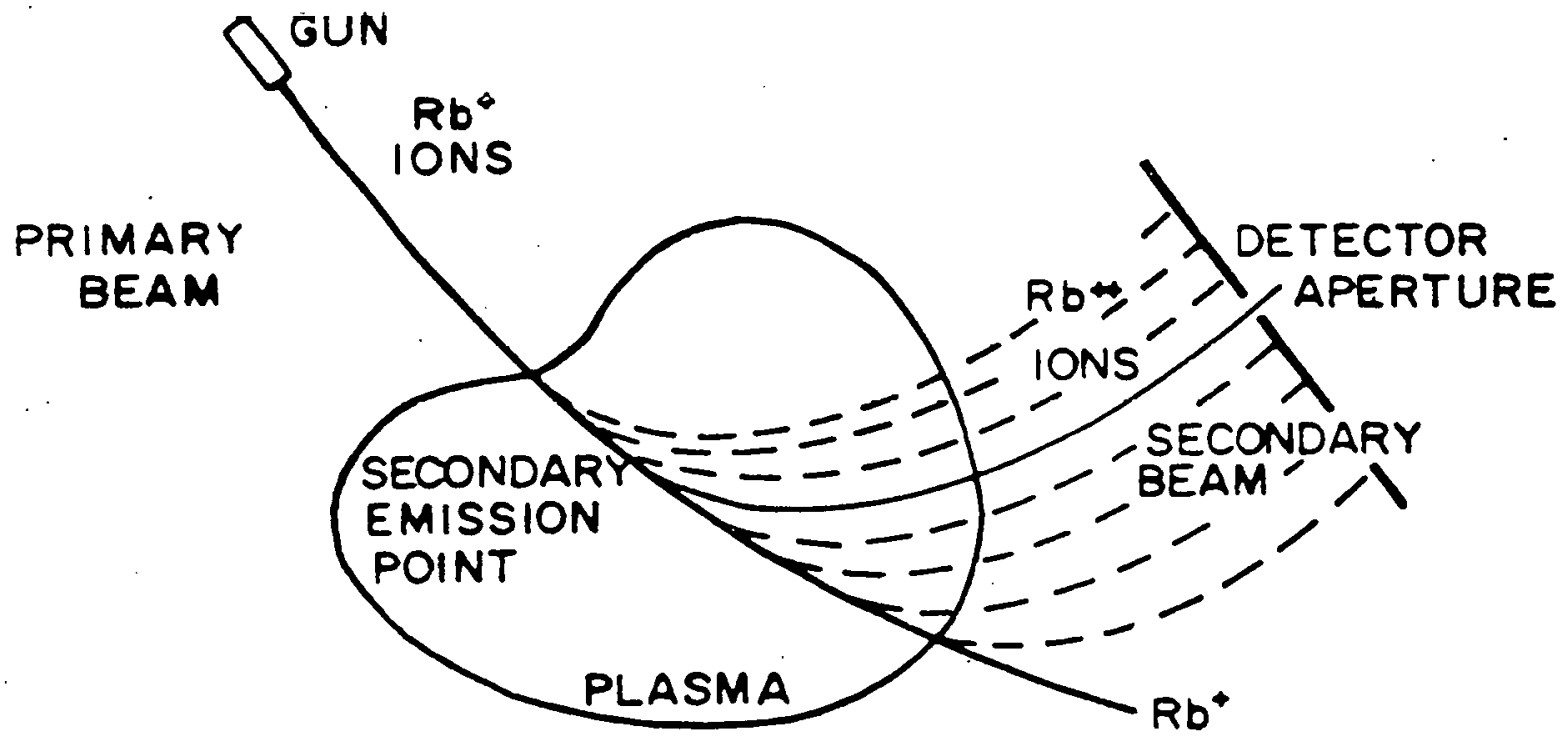

Fig. 1 Basic Concept of Heavy Ion Beam Probing 
we are creating a test particle with known properties at a well defined point in the plasma and by measuring the properties of the test particle after it leaves the plasma, we can extract considerable information about the p.lasma environment where it was created.

The intensity of the secondary ion current is given by

$$
I_{s}=2 I_{p} \frac{\langle\sigma V\rangle}{V_{b}} n \ell
$$

where $n$ is the plasma electron density, $\ell$ is the length of primary beam path that is being sampled, $v$ is the relative velocity between the beam ion and the plasma electron, and $V_{b}$ is the beam velocity. The brackets denote averaging over the electron velocity distribution function. In general the ionization energy is supplied by the electron thermal energy. This is most easily understood by transforming to a coordinate system that is moving with the beam velocity which typically is of the order of $10^{7} \mathrm{~cm} / \mathrm{sec}$. An electron that is a rest in the laboratory frame will have a velocity of $10^{7}$ in the moving frame which corresponds to an energy of the order of $0.1 \mathrm{eV}$. This is much less than the ionization threshold. Consequently, the effective cross section is a function of the electron temperature, and equation (1) can be rewritten as:

$$
I_{s} / I_{p}=\gamma \cap F\left(T_{e}\right)
$$

where $\gamma$ is a geometric factor and $F\left(T_{e}\right)$ is the effective cross section for the particular reaction and beam velocity that is being used. If we probe the same point in the plasma with two different reactions that have different 
$F\left(T_{e}\right)$ values then it is possible to separately evaluate $n$ and $T_{e}$. This is discussed in more detail in Section $V$.

The energy of the primary beam as it enters the plasma is eV $V_{g}$ where $V_{g}$ is the ion gun voltage. When it reaches the point $p$ in the plasma, it will have an energy of $\mathrm{e}\left(\mathrm{V}_{\mathrm{g}}-\phi\right)$ where $\phi$ is the space potential at the point $p$. A secondary ion created at this point will gain an energy of 2e $\phi$ on leaving the plasma and end up with a net energy of $e\left(V_{g}+\phi\right)$. By comparing the energy difference between the primariry and secondary furls, we obtain a direct measurement of the space potential at the point $p$.

The trajectories of the primary and secondary ions are uniquely determined by the injection parameters and the magnetic field. If the plasma has a finite beta, then the trajectories will differ from the vaccum field trajectories. By measuring the change in trajectories, it is possible to obtain information about beta. This is an integrated effect along the beam trajectory so it is different in character from the $n, T_{e}$, and $\phi$ determinations which are essential point measurements. Similarly, it is conceivable to use this technique to study the magnetic field structure, due for example to plasma currents, but again it is an integrated measurement and very difficult to unravel.

Typical operating parameters for present day beam probe systems are beam energies in the range from 10 to $100 \mathrm{KeV}$, beam currents of the order of 10 to 50 microamps. Secondary ion signals range from 1 to a few hundred nanoamps. Space resolution is of the order of 0.1 to $1.0 \mathrm{~cm}^{3}$ and time resolution is of the order of 10 microseconds. The $n F\left(T_{e}\right)$ and $\phi$ signals can be measured simultaneously and the measurements are continuous in time. 
It has been tacitly assumed that the magnetic field has only a single component, which we will choose as the $z$ component. For this case the beam trajectories are confined to the $x-y$ planes as illustrated in Fig. 1. In general this is not true, but for a very large number of systems (tokamaks, EBT's, simple mirrors), it is a very good first order approximation. We will continue to make this basic assumption for the time being, but will consider the effects of multi-component magnetic fields in Sections VII and VIII.

\section{MAPPING THE PLASMA}

In the previous section, the general concept of beam probe was presented and a brief description of the types of measurements were described from the point of view of looking at a small preselected sample volume in the plasma. It is obvious that simultaneous measurements could be made at a number of points on the primary trajectory by installing multiple detectors in the fan of secondary ions. The use of multiple detectors is cumbersome and unnecessary. It is possible to map the complete plasma cross-section using a fixed single detector by sweeping the beam injection angle and energy. This is illustrated in rig. 2 which shows two primary beam paths having different injection angles. For the upper primary path, secondary ions created at the location marked $E_{3}$ will enter the detector and for the lower path, the observation point is marked $E_{4}$. If we sweep the injection angle with the sweep plates as indicated, then we would measure the plasma profile along the curved line $E_{3}-E_{4}$. This is referred to as the detector line. Note that secondaries created at the points $E_{1}$ and $E_{2}$ do not enter the 


\section{BEAM PROBING SYSTEM}

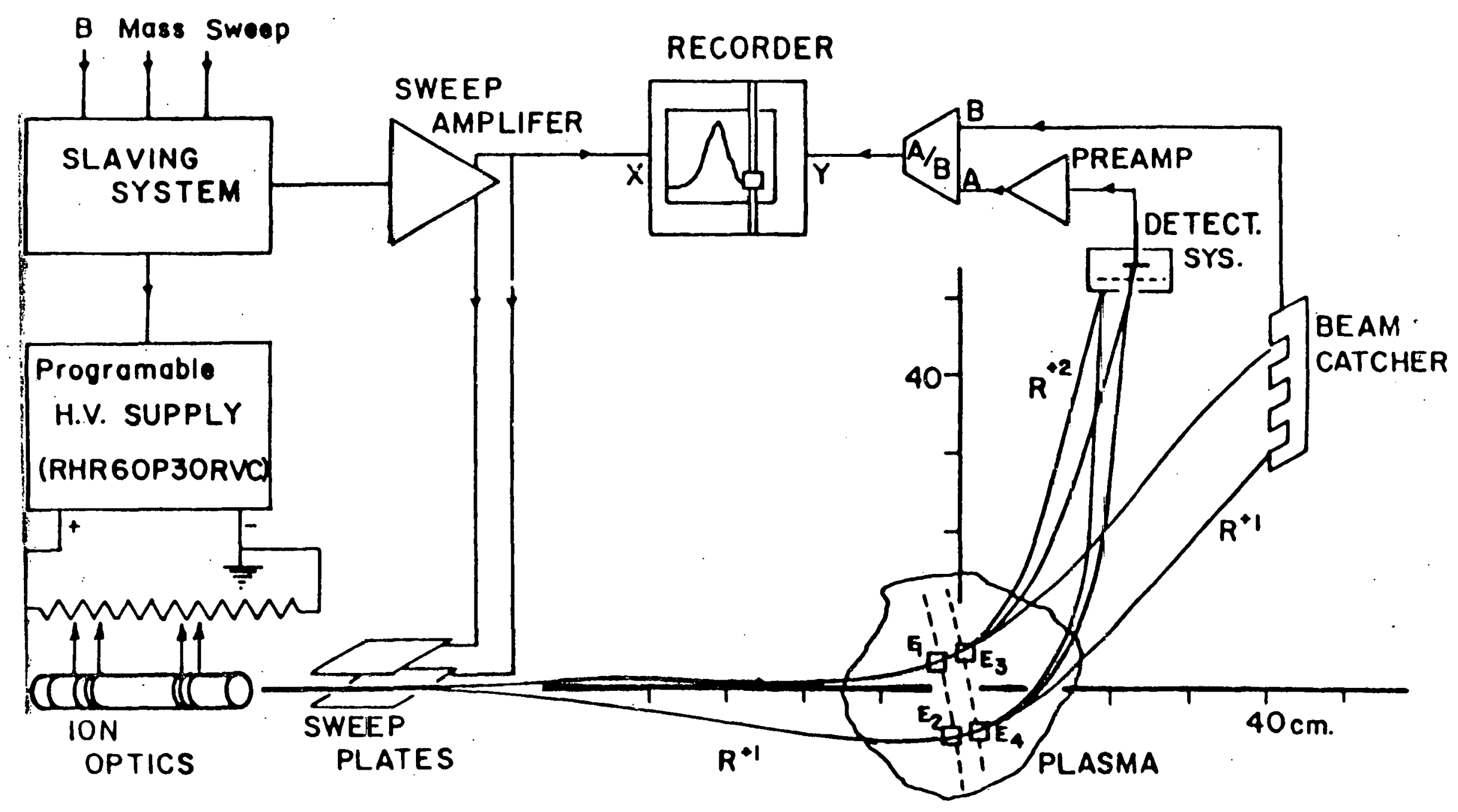

Fig. 2 Simplified schematic 
detector aperature. If we increase the beam energy slightly (i.e. stiffen the beam and increase the radius of curvature) then secondarires from $E_{1}$ and $E_{2}$ would enter the detector but those from $E_{3}$ and $E_{4}$ would miss. Actually when we increase the beam energy we will also change the primary trajectories slightly, but this change is too small to be observable in Fig. 2. We can generate a new detector line at the higher beam energy which is denoted by $E_{1}-E_{2}$. Increasing the beam energy moves the detector line towards the ion gun and decreasing the energy moves it away from the gun. Thus by proper choice of beam energy and injection angle, it is possible to look at any preselected point in the plasma with a single fixed detector. The combination of beam energy and injection angle generates a detection grid spanning the plasma cross section. . There is a one-to-one correspondence between the beam energy-injection angle and the $x-y$ coordinate of the sample volume.

Figure 2 also illustrates in simplified schematic form all of the basic elements of a heavy ion beam probe system. Starting in the upper left, there is a slaving system which receives as inputs the value of the magnetic field, the mass of the ion being used and the desired sweep function. The slaving system sends out signals to the sweep amplifier and the programmable high voltage supply used to drive the ion gun. This determines the particle trajectories and the observation point in the plasma. There is a beam catcher to monitor the primary beam current and a secondary ion detector. The signals are then fed into an appropriate recording system which also receives as input the location of the observation point. The output is a map of the desired plasma parameters as a function of position in the plasma. 
This simplified schematic omits a lot of necessary, but confusing detail and actual systems are much more sophisticated and complex. This is illustrated in Fig. 3, which shows a detailed schematic of the heavy ion beam probe system installed on the center cell of TMX.

\section{SPACE RESOLUTION}

The space resolution is determined by the diameter of the primary beam and the width of the detector aperature. Consider first the situation where the primary beam has a well defined circular cross section with uniform current density and the detector lines from the two edges of the detector aperature are straight and parallel across the beam diameter. The projection of this scenario on to the $x-y$ plane is illustrated in Fig. 4 where the 2 lines labeled $b$ represent the beam and the 2 lines labeled $d$ represent the detector lines. Actually we should refer to detector planes as it is assumed that the dimension of the detector aperature in the $z$ direction is much larger than the beam dimensions. The sample volume then consists of a circular cylinder with parallel end planes that cut the cylinder at an angle $\theta$ with respect to the cylindrical axis. The size of this sample volume is $\pi r^{2} \ell$. In the $x-y$ projection the sample volume cross-section is a roughly parallelogram with acute angle $\theta$. The volume is not a function of $\theta$, but it should be noted that as $\theta$ gets smaller, the major diagonal of the parallelogram increases and the minor one decreases. In the limit one goes to infinite as the other goes to zero maintaining the volume constant. A long narrow resolution volume is not a desirable situation since it is desirable to have all dimensions of the resolution volume smaller than any plasma spatial gradients. The ideal situation is when $\theta$ is equal to $90^{\circ}$ 


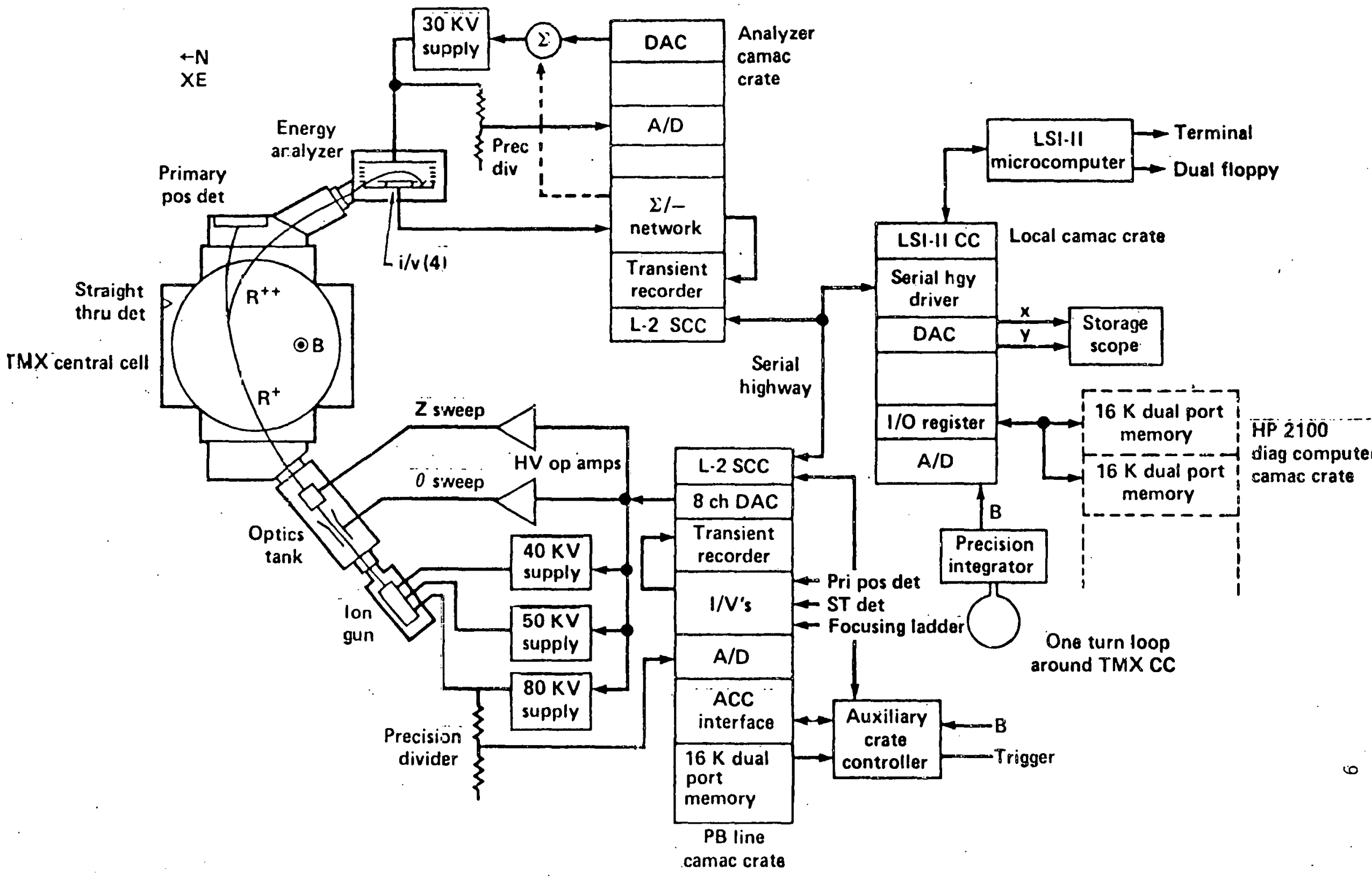

Fig. 3 Deticiled schematic of the beam probe system installed on TMX 


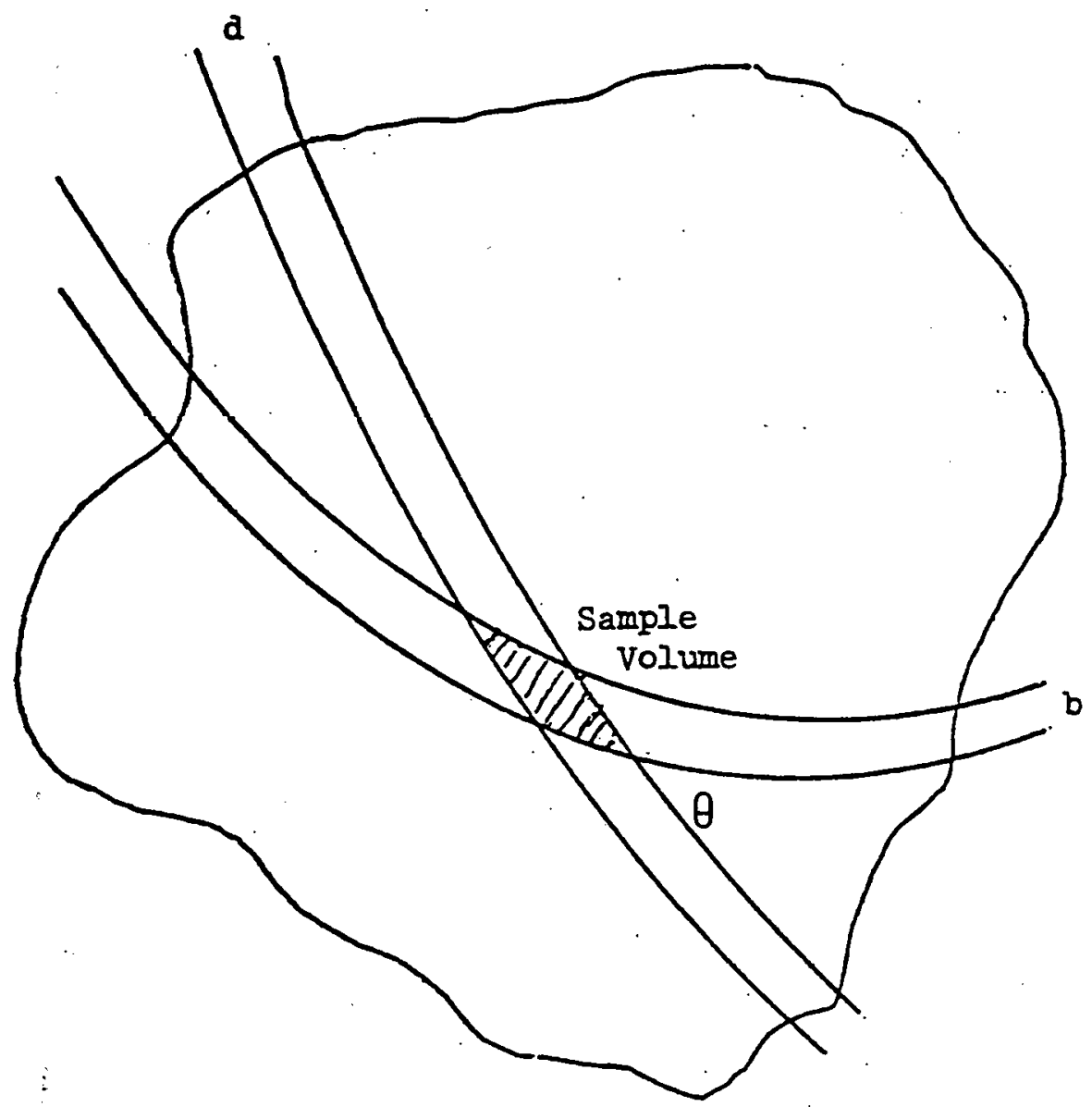

Fig. 4 Projection of the sample volume in the $x-y$ plane 
and the sample length is equal to the beam diameter. This would correspond to a rectangular grid of detector lines and beam paths. In general this ideal rectangular grid cannot be maintained over the full plasma cross section. One of the major concerns in designing a beam probe system for a given plasma device is trying to optimize the detector grid. The detection grid is determined by the injection point, magnetic field shape and detector location, and is generally constrained by the available entrance and exit ports. Figure 5 shows the detector grid for the beam probe system in operation on EBT-1/S at ORNL. The nearly vertical lines correspond to beam paths at constant beam energy, but different sweep voltages between \pm 500 volts. The curved lines labeled from 10 to 24 correspond to detector lines for various beam energies. Note that near the center of the system there is nearly a rectangular grid pattern, but it gets strongly distorted as you move out from the center, particularly near the top of the plasma. For this particular case not only does the shape of the sample volume change with position, but the size of the volume changes also and this must be corrected for when making $n F\left(T_{e}\right)$ measurements.

The parallel beam-parallel detector line is a good first order approximation to the sample volume. A more detailed analysis has to take into consideration that the beam does not have uniform current density, is not parallel and is not monergetic. We will restrict our discussion to the sample area in the $x-y$ plane, but extension to include the $z$ dimension is straightforward. Let $j(r, v, E)$ represent the primary beam current density at position $r$, having a velocity $v$ and energy $E$. For any point, $r$, in the plasma there is a range of primary beam energies and velocities that will 


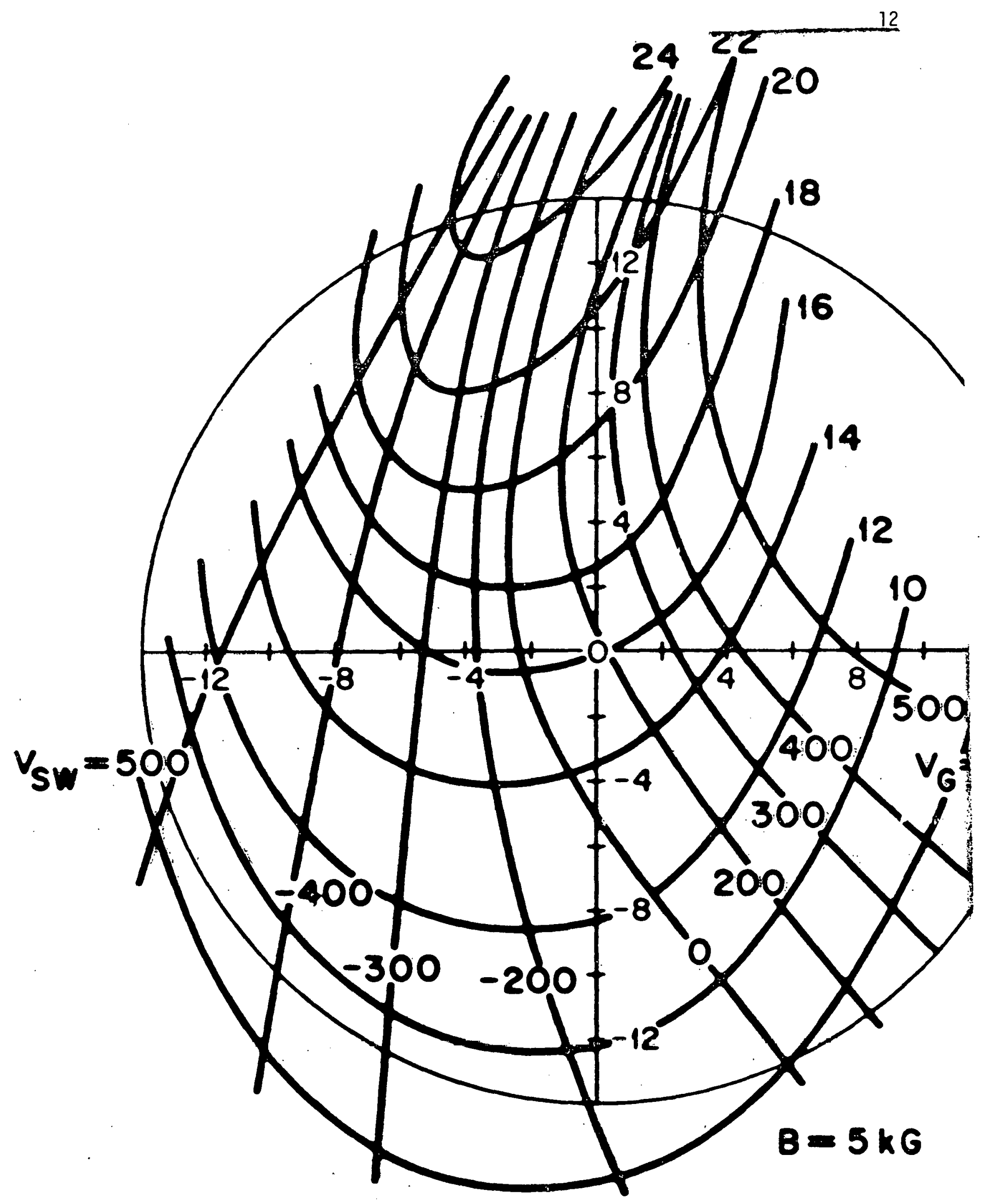


yield secondary ions that hit the detector aperature. This can be represented by a probability function $P(r, v, E)$. For a given set of parameters $(r, v, E)$, this probability function is either zero or one (i.e. the secondary ion either enters the aperature or it does not). The actual sample volume is then obtained by a convolution of $P(r, v, E)$ and $j(r, v, E)$. This has to be evaluated for each specific experiment, but in general what it does is to give rounded edges rather than the sharp boundaries predicted by the parallel beam-parallel detector line approximation.

The fan of secondary ions (see Fig. 1) leaving the plasma will in general be diverging. For this case the length of sample volume for a given width detector aperature depends on the location of the detector. If the detector is moved further away from the plasma it will observe a smaller length of the primary beam. The ratio of the aperature width to the sample length is referred to as the magnification of the system. So the detector location not only effects the shape of the detection grid, but also determines the sample volume for a given aperature. For large aspect ratio toroidal systems, such as tokamaks, there is a lens effect due to the $1 / R$ variation of the toroidal field. In this situation the fan of secondary ions can converge as they leave the plasma and if this is not taken into consideration it can lead to large sample volumes spoiling the space resolution. This is usually avoided by designing the system such that the effective sweep point for the primary beam is inside the focal length of the magnetic lens. For a mirror type magnetic field that decreases in all radial directions as you move out from the center of the plasma, there is a strong divergence of the secondary ions. It acts more like a concave lens. This 
is what causes the strong distortion in the EBT-1/S grid shown in Fig. 5 . Ideally one would like a weakly diverging fan of secondary ions that is roughly the same for all primary beam injection angles. This situation can generally be obtained when the radius of the magnetic field is much larger than the plasma radius. This is the situation for the center cell of TMX and the detection grid for this system is shown in Fig. 6. Note that this grid is nearly uniform over most of the plasma region. It is not rectuangular, however, and this is due to the constraints imposed by the existing port structures. If we had been allowed to specify the ports; then we could have designed a system that would have provided a nearly uniform rectangular grid over the full plasma cross section.

\section{SPACE POTENTIAL MEASUREMENTS}

Probably the most important contribution of ion beam probing is the direct measurement of the plasma space potential. As noted in Sect. I, the space potential is determined by measuring the difference in energy between the primary and secondary ions which is just equal to eф. Typically it is desired to know $\phi$ to ten volts or less and typical beam energies are in the range of 10 to $100 \mathrm{KeV}$. Consequently, we need to measure an energy difference between the primaries and secondaries to one part in $10^{-3}$ or $10^{-4}$. This is accomplished by using a modified parallel plate electrostatic energy analyzer. The modification consists of replacing the conventional narrow exit aperature with a wide aperature and using split plate detectors. The wide exit aperature assures that all of the beam entering the entrance slit will hit the split plate detectors. The difference signal between the two 
Ion: $\mathrm{K}$

Energy: $12.4-18.4 \mathrm{kV}$

Azimuth: $121-137 \mathrm{deg}$

Polar: $90 \mathrm{deg}$

$X O: 70.0 \mathrm{~cm}$

YO: $-140.0 \mathrm{~cm}$

XDET: $125.0 \mathrm{~cm}$

YDET: $171.0 \mathrm{~cm}$

Plasma radius: $30 \mathrm{~cm}$

$B: \quad 500.0$ gauss

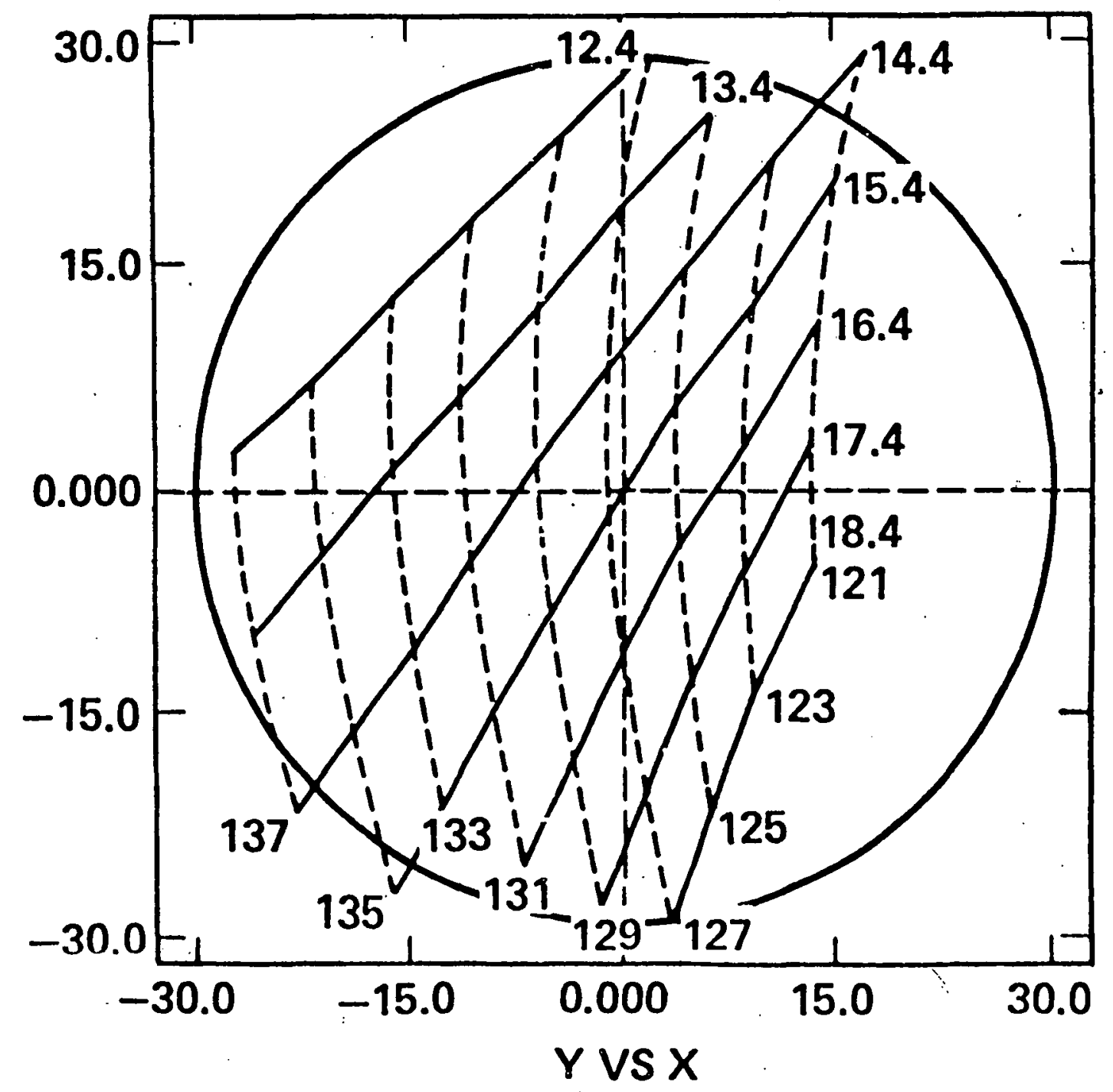


split plates is then amplified and fed back to the top plate of the analyzer which forces the beam to be provided equal currents to the two split plates. If the system is set up correctly, the feedback voltage is directly proportional to the space potential. At the same time, the signals from the two split plates are added together to provide a measure of $n F\left(T_{e}\right)$. This technique is illustrated schematically in Fig. 7 and an isometric diagram of an analyzer is shown in Fig. 8.

In a typical installation, a D.C. voltage that is slaved to the gun energy is connected to the top plate of the analyzer and the feedback signal is A.C. coupled on top of the D.C. This feedback controlled analyzer can provide a sensitivity of the order of $10^{-4} \mathrm{~V}_{\mathrm{g}}$. The system is calibrated by directing the primary beam into the analyzer and adjusting the slaving system to center the signal on the two split plates with zero feedback voltage. A precise gain of $1 / 2$ amplifier is then inserted into the feedback loop to compensate for the $2^{+}$charge of the secondary ions.

The voltage required to center the beam on the split plates is a function entrance angle between the beam path and the analyzer ground plane. As the observation point is scanned across the plasma this entrance angle changes and has to be taken into account when extracting the space potential. In principal, the entrance angle can be determined from trajectory calculations and then used to correct the data. In practice, however, we prefer to reduce the sensitivity to entrance angle to the point where the correction can be ignored. The angular characteristics of the analyzer are determined by the ratio of the separation between the entrance aperature and the split detectors to the sum of the distance of the entrance aperature and 


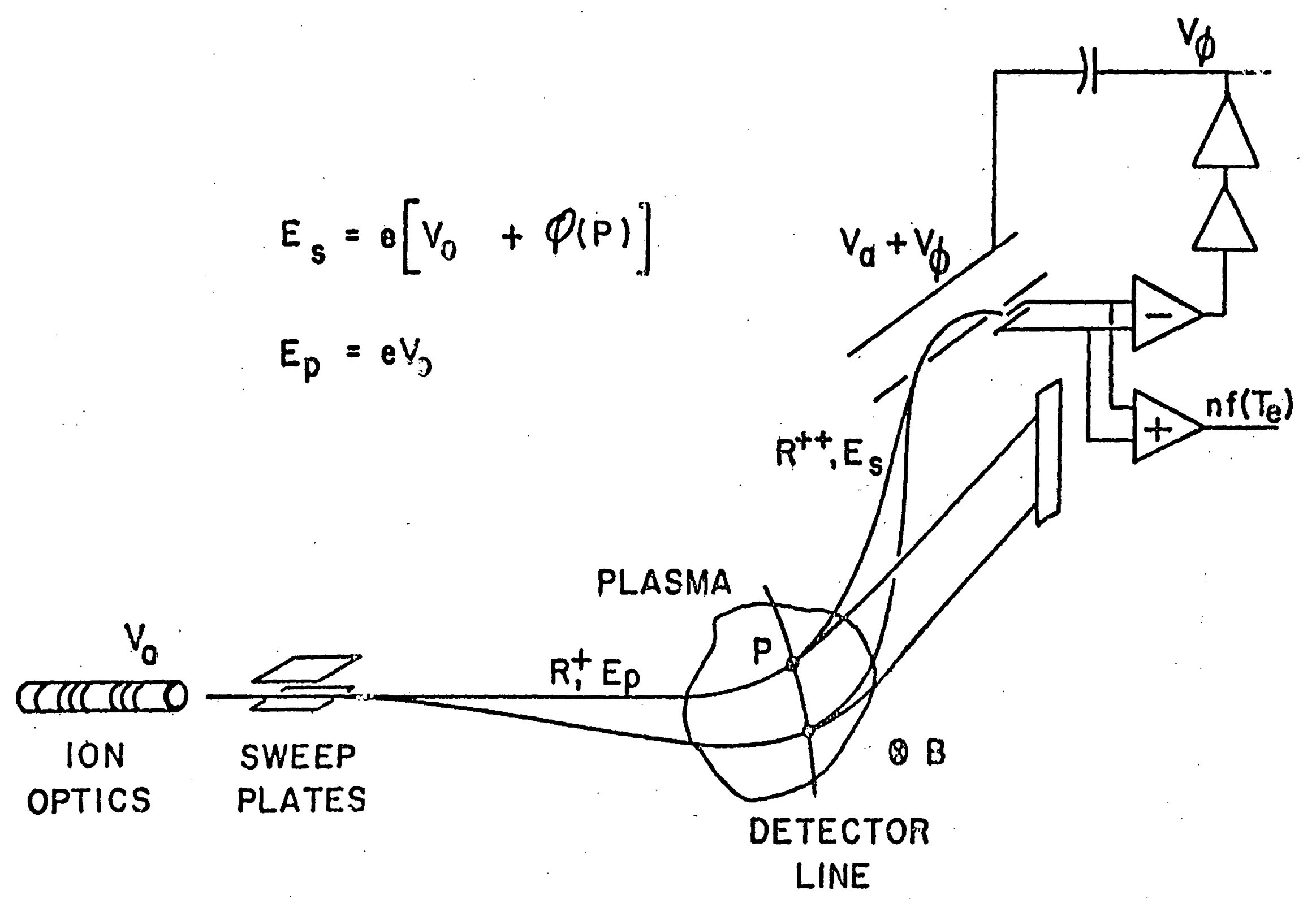

Fig. 7 Simplified schematic in illustrating the space potential measurement. 


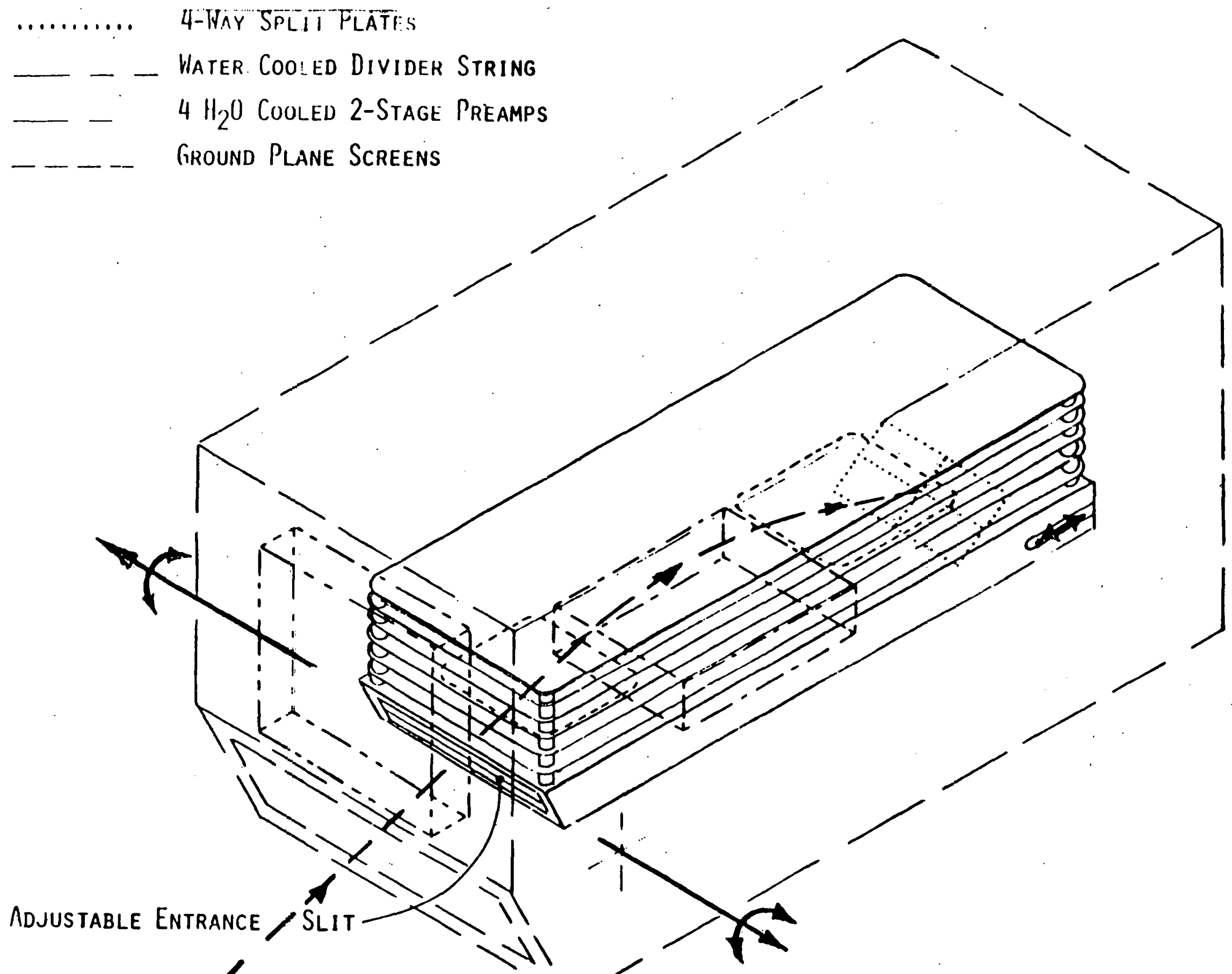

Fig. 8 Isometric drawing of electrostatic energy
analyzer 
split detector below the ground plane of the analyzer. A typical set of angular correction curves is shown in Fig. 9. These curves were all taken on the same analyzer. The central curve marked $4+$ corresponds to a nominal separation between the entrance slit and detector of $25.4 \mathrm{~cm}$ which was the design value for this analyzer. The top curve is for a separation of 24.4 $\mathrm{cm}$ and the bottom curve for $26.4 \mathrm{~cm}$. If we operate with the upper curve, we have a $10^{\circ}$ window with less than \pm 12 volt correction; or we could use the center curve and obtain a $20^{\circ}$ window with less than \pm 35 volt correction. On the newest analyzers, this separation between entrance aperature and split detectors is adjustable from outside the vacuum system. This permits the angular correction curve to be changed or adjusted without opening up the system. Two other things should be noted with regard to Fig. 9: (1) The angular position has an arbitrary zero and the position noted as $15^{\circ}$ actually corresponds to an entrance angle of approximately $30^{\circ}$. (2) There is a fairly large change in analyzer voltage as the separation is changed. This is due to a change in the geometric gain of the analyzer which is defined as the ratio of top plate voltage to beam energy when the beam is center on the split plates. As you decrease the separation between the entrance aperature and detector, then it is necessary to increase the voltage to center the beam on the detector.

Energy resolution with the feedback analyzer is essentially determined by the noise level of the first stage amplifier instead of the aperature widths. The two split plate signals cannot be nulled out to any greater precision than the amplifier noise. In many instances the noise pickup on the split plate detectors is larger than the amplifier noise and 


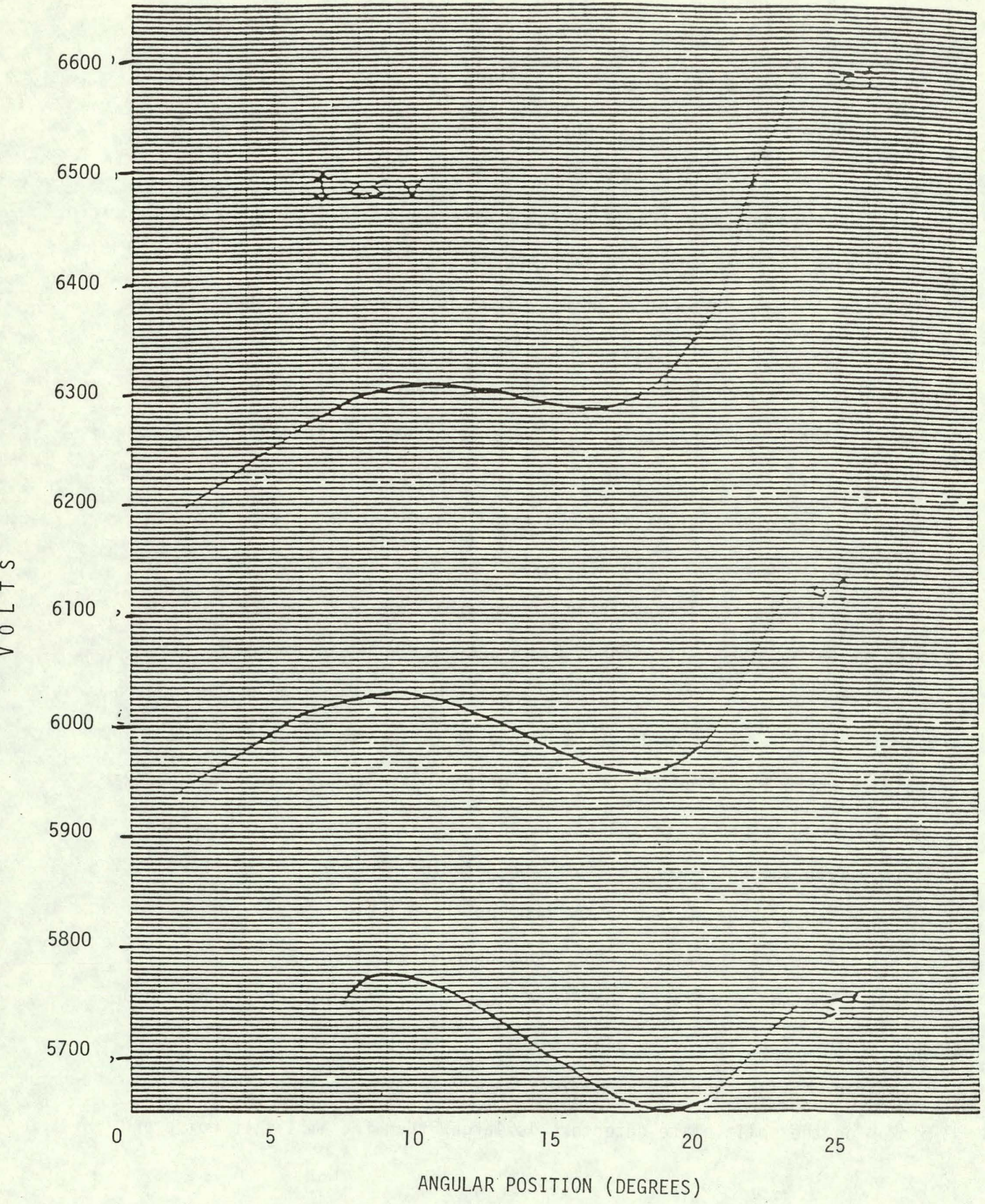

Fig. 9 Angular Correction Function for Energy Analyzer 
consequently, this sets the limit on the resolution. It should also be noted that we are making a relative measurement between the primary beam energy and the secondary beam energy. As stated earlier, this can generally be done to about 1 part in $10^{4}$, but absolute measurements with respect to machine ground is normally. only about 1 part in $10^{3}$. In order to obtain this type of resolution it is extremely important that there is very little energy spread in the primary beam. To obtain this low energy spread thermionic ion sources are used. All beam probe measurements to date have been make with alkali metal ion sources ( $L i, N a, K, R b, C s, T \ell$ ) since it is relatively easy to make thermal ion sources for these materials. This is accomplished by loading a "zeolite" with the appropriate alkali metal and then heating it to approximately $1000^{\circ} \mathrm{C}$ and applying an extraction voltage. This will supply ion beam currents of the order of 10 to 100 $\mu \mathrm{amps} / \mathrm{cm}^{2}$.

The space potential that is measured is a weighted average over the sample volume. If the $n F\left(T_{e}\right)$ signal is uniform over the sample volume, then the weighting for the potential measurement is also uniform. If on the other hand, then $n F\left(T_{e}\right)$ is not uniform, then it will weight the averaging process since we are balancing the current signals to the two split.plates. We now normally split the detector plates four-ways insteady of just two (i.e. left-right as well as up-down). This is done to facilitate alignment of the system. Recall that we want the gun, beam trajectories and analyzer center line to all lie in the $x-y$ plane. This is not necessarily easy to accomplish to high precision by pure mechanical means. By splitting the detector plates in the left-right direction and installing $z$ steering 
plates on the primary beam line, we can electrically adjust the system so that the secondary beam is $z$ centered in the analyzer.

V. $n F\left(T_{e}\right)$ MEASUREMENTS

The sum current on the split plate detectors provide a measurement of $n F\left(T_{e}\right)$. If either $n$ or $T_{e}$ is known, then it is possible to extract the other parameter: Recall that $F\left(T_{e}\right)$ represents the effective cross section for the particular beam being used averaged over the electron velocity distribution. Figure 10 is a plot of $F\left(T_{e}\right)$ for a $20 \mathrm{KeV} \mathrm{Rb}^{+}$beam as a function of electron temperature. This is a typical beam parameter for the RENTOR tokamak. Above about $100 \mathrm{eV}, F\left(T_{e}\right)$ is fairly insensitive to $T_{e}$; but if the curve had been extended to higher electron temperatures, it would have shown that $F\left(T_{e}\right)$ slowly decreases. If it is known that $T_{e}$ is in the $100-200 \mathrm{eV}$ range, then the sum signal is directly proportional to the plasma density and the density can be evaluated.

If we want to use the beam probe to separately evaluate $n$ and $T_{e}$, then we need to look at the same point in the plasma with two different reactions that have different $F\left(T_{e}\right)$ values. One way to do this is to use two different ion species, say $\mathrm{Na}^{+}$and $\mathrm{K}^{+}$and adjust their momentum so that they follow the same trajectories. We can then take the ratio of the normalized secondary currents, the density term will drop out, and $T_{e}$ can be evaluated. This assumes that the density does not change between the two measurements. The easiest way to accomplish this is to load the "zeolite" with both ion species, then change the gun energy so that you look at the same point with first one and then the other ion. This technique is only 
useful for relatively low temperature plasmas, say less than $100 \mathrm{eV}$. Above this temperature the $F\left(T_{e}\right)$ ratios are relatively insensitive to $T_{e}$ since they are both fairly constant.

So far we have talked about impact ionization reactions that generate $2^{+}$secondary ions. Impact ionization collisions can also produce $3^{+}, 4^{+}$, and higher charge state secondary ions. This is illustrated in Fig. 10 which shows the generation of $2^{+}, 3^{+}$, and $4^{+}$signals on EBT. This data was generated by holding the primary beam parameters fixed and scanning the analyzer voltage. It should be noted that these signals are not coming from the same point in the plasma, but the system can be set up to look at the higher charged state secondaries from the same location used for the $2^{+}$ signal. The effective cross section for generating the higher charge states is obviously different then the cross section for generating $2^{+}$and conse- . quently the $3^{+}-2^{+}$ratio or $4^{+}-2^{+}$, etc., can be used to evaluate $T_{e}$. There are a number of problems in implementing this technique. The two major ones are that even when looking at the same point in the plasma, the sample volumes are not the same due to the difference in trajectories of various secondary ions and the multiple ionization cross sections are not well known. At the present time we do not recommend this technique for trying to obtain an absolute measure of $\mathrm{T}_{e}$, but it is very useful in continuously monitoring relative changes in $T_{e}$. The preferred procedure would be to use Thomson Scattering to obtain an absolute value combined with the $3^{+}-2^{+}$ratio to provide a monitor of relative changes. It is interesting to note that the higher charge states can also be used to provide a potential measurement, and this is a convenient way to confirm the $2^{+}$potential measurements. 
Relative Intensity of $2^{+}, 3^{+}$, and $4^{+}$Signals

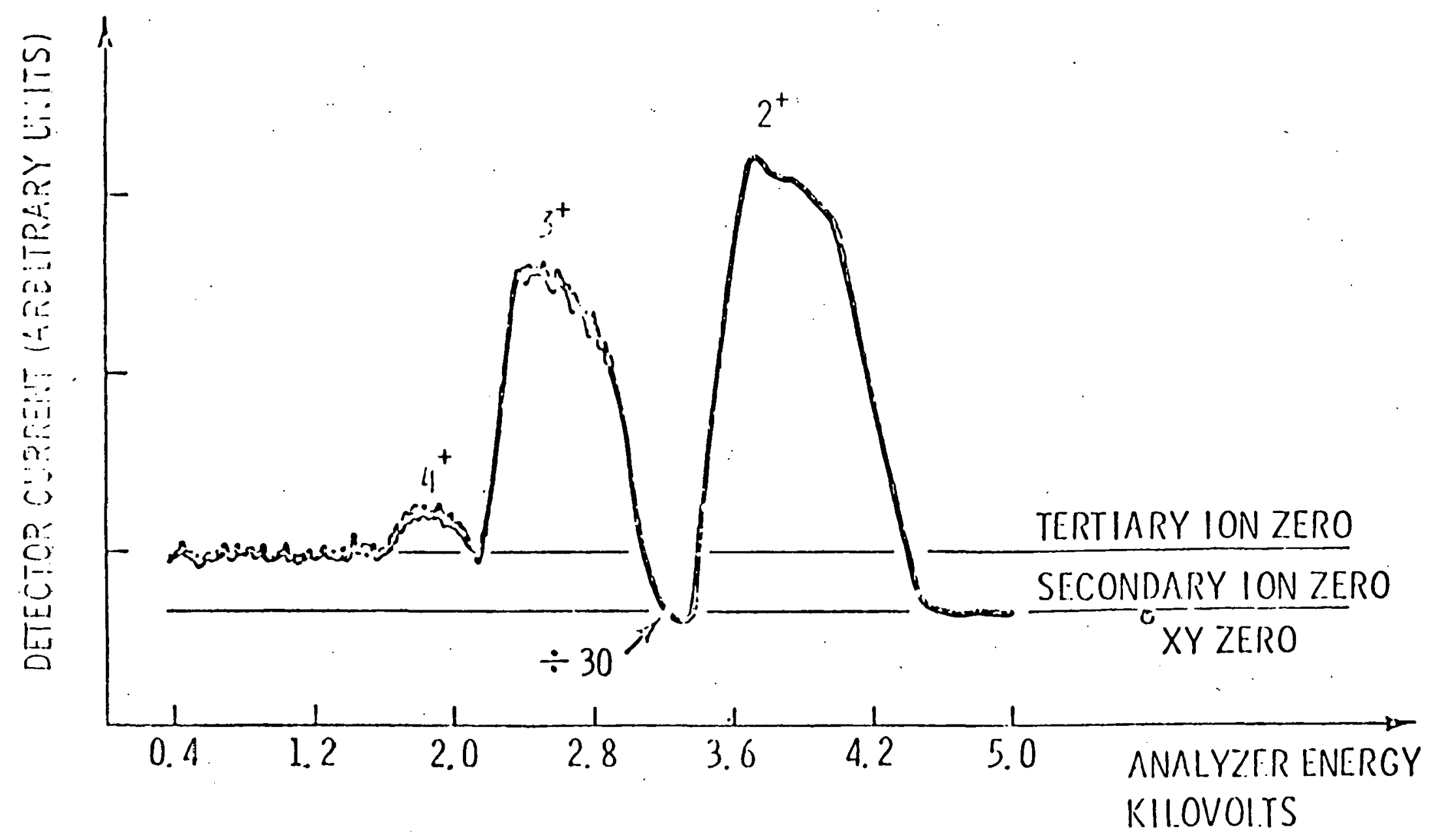

Fig. 10 EBT data showing the production of $3^{+}$and $4^{+}$secondary ions 
As noted in Sect. III the sample volume in general is not uniform over the plasma cross-section. This has to be taken into account in evaluating the $n F\left(T_{e}\right)$ signals. Usually there are various wire screens and meshes on both the primary and secondary beam lines (for example over the entrance and exit aperatures of the analyzer and sometimes between the plasma chamber and the gun and detector chambers). Transmission through these screens may be a function of the observation point and this has to be evaluated for each specific system.

In the evaluation of $\mathrm{nF}\left(\mathrm{T}_{e}\right)$, the signal must be normalized to the primary beam current delivered to the sample volume. Generally the primary beam current changes as the beam energy is scanned and monitoring the primary beam current during plasma operation is fairly difficult. This difficulty is usually due to the lack of exit ports for the priamry beam and, consequently, the monitor is placed in the plasma chamber. This results in large noise pickup during plasma operation which swamps the beam signal. The usual procedure is to monitor the primary current before and after a measurement and use this calibration to normalize the secondary signal. Frequently there is appreciable attenuation of the primary beam as it penetrates the plasma and this, too, must be accounted for in evaluating the measurements. This can be done by using the data from the outer edges of the plasma where there is no appreciable beam attenuation to calculate the primary beam intensity delivered to the next zone. This procedure is then repeated to find the intensity delivered to the next zone. 
VI. TIME RESOLUTION

A. $n F\left(T_{e}\right)$ Measurements

The ultimate limitation on the time resolution for this measure is the time required to collect enough secondary ions to provide the desired statistical accuracy. If $3 \%$ statistical accuracy is desired, then it is necessary to collect 1000 secondary ions. If the secondary ion current was of the order of a nanoamp, then this time would be of the order of $10^{-10}$ sec. In practice, however, the time resolution is set by the state-of-theart electronics used to detector the secondary ion signal. The best amplifiers presently available have about 1 nanoamp sensitivity at a bandwidth of the order of $1 \mathrm{MHz}$. The bandwidth can be increased by increasing the amplitude of the secondary ion current. For most systems, the secondary ion current is in the 10 to 100 nanoamp range and $1 \mathrm{MHz}$ has been more than adequate for all the measurements that have been presently attempted. In fact we frequently cut the bandwidth back in order to improve the signalto-noise ratio.

Increasing the primary current increases the secondary signal, and this would permit an increase in the time resolution. It should be possible to increase the primary current density substantially above present operating levels, but very little attention has been devoted to this since the present systems have been adequate for most purposes. Another way to increase the. secondary signal level is to increase the size of the sample volume. Thus it is possible to trade off space resolution for time resolution. 
Beam probing has been used to study MHD instabilities, but generally these lie in the frequency range below $100 \mathrm{KHz}$. Presently avajlable time resolution is adequate for these studies. The next class of instabilities would have frequency in the range of the ion cyclotron frequency which for typical systems would be in the 10's of MHz range. It would take considerable developmental work to obtain this short a time resolution and very little effort has been devoted to trying to accomplish this.

\section{B. $\phi$ Measurements}

The basic limitation on the space potential measurements is the transit time of the secondary ions through the electrostatic energy analyzer. Typically this is of the order of 0.1 to $1.0 \mathrm{microsec}$. In practice however, the time resolution is limited by the frequency response of the very high gain amplifier string used in the feedback loop. The transimpedance of this amplifier string is of the order of $10^{11}$ ohms and it is very difficult to obtain this order of transimpedance with high frequency response. The best amplifier systems that have been used to date have had a flat response out to $50 \mathrm{KHz}$ and a useful range out to approximately $100 \mathrm{KHz}$. Increasing the signal strength would also reduce the demands on the feedback amplifiers, and this could be more important for the space potential measurements than for $n F\left(T_{e}\right)$.

Recently we have started to process the space potential off line rather than closing the analog feedback loop. We record the signal from both split plates and then calculate the voltage that would be required to center the beam. We have just started to do this on TMX and RENTOR and do 
not yet have sufficient information to make a comparison between on line and off line sensitivity. Off line processing should work well as long as the space potential variation is small enough so that it doesn't drive the signal completely on to one plate or the other. One possible way we are thinking about to avoid this is to use a slow feedback loop to keep the beam reasonably centered on the split plates and then do the final processing off line. With off line processing it should be possible to increase the frequency response of the $\phi$ measurements to the same order of magnitude as that for the $n F\left(T_{e}\right)$ measurements. The transit time through the analyzer would not set a limitation since the field in the analyzer would remain constant.

\section{BETA AND MAGNETIC FIELD MEASUREMENTS}

Up to now we have assumed that we could calculate the beam trajectories to any degree of accuracy. This is true if we know the magnetic field, but if the plasma distorts the field in any manner or if the potential is so high that it distorts the trajectories, then this assumption is no longer valid. If the plasma beta appreciably reduces the field from the vacuum field value, then we will no longer be able to predict the trajectories. In the limit of $\beta=1$ the beam will travel in a straight line through the plasma, and we will no longer have any space resolution (i.e. it will be an integrated measurement). If $B$ is finite, but less than unity we will still be able to make the measurements but we will loose track of the location of the sample volume. The finite beta will also cause the primary beam to follow a different trajectory through the plasma and strike the primary beam detector at a different 
location. By monitoring the location of the primary beam and mapping the sample volume across the plasma cross section, it should be possible to use a converging iteration calculation of the beam trajectories that will provide a map of beta. This has not been done, but it appears to be a possible experiment on TMX. Figure 11 shows the detection grid on TMX for an assumed square well $\beta$ of 0.5 and a gaussian potential profile with a peak value of $1000 \mathrm{~V}$. This should be compared with Fig. 6 which is the detection grid for the vacuum field case.

If there is current flow in the plasma that generates a magnetic field such as the poloidal field in a tokamak, then it may be possible to measure this field. On a tokamak, the poloidal field imparts some $z$ or toroidal momentum to the beam. If the $z$ displacement or the $z$ velocity of the secondary ions is measured at the detector, then it can be shown that in cylindrical approximation they are related to the poloidal magnetic field at the creation radius by

$$
\begin{aligned}
& \frac{d \dot{z}_{D}}{d r_{e}}=k B_{\theta}\left(r_{e}\right) \\
& \frac{d z_{D}}{d r_{e}}=k^{\prime} B_{\theta}\left(r_{e}\right)+\text { Higher Order Terms }
\end{aligned}
$$

where $k$ and $k^{\prime}$ are geometric constants. More generally it can be shown that if there is an ignorable coordinate of the vector potential, then the other two components can be evaluated. For ideal tokamaks, the toroidal coordinate is ignorable. 
Ion: $\mathrm{K}$

Energy: $12.1-13.2 \mathrm{kV}$

Azimuth: $122-134 \mathrm{deg}$

Polar: 90 deg

$X O: 70.0 \mathrm{~cm}$

YO: $-140.0 \mathrm{~cm}$

XDET: $125.0 \mathrm{~cm}$

YDET: $171.0 \mathrm{~cm}$

Plasma radius: $30.0 \mathrm{~cm}$

$B: \quad 500.0$ gauss

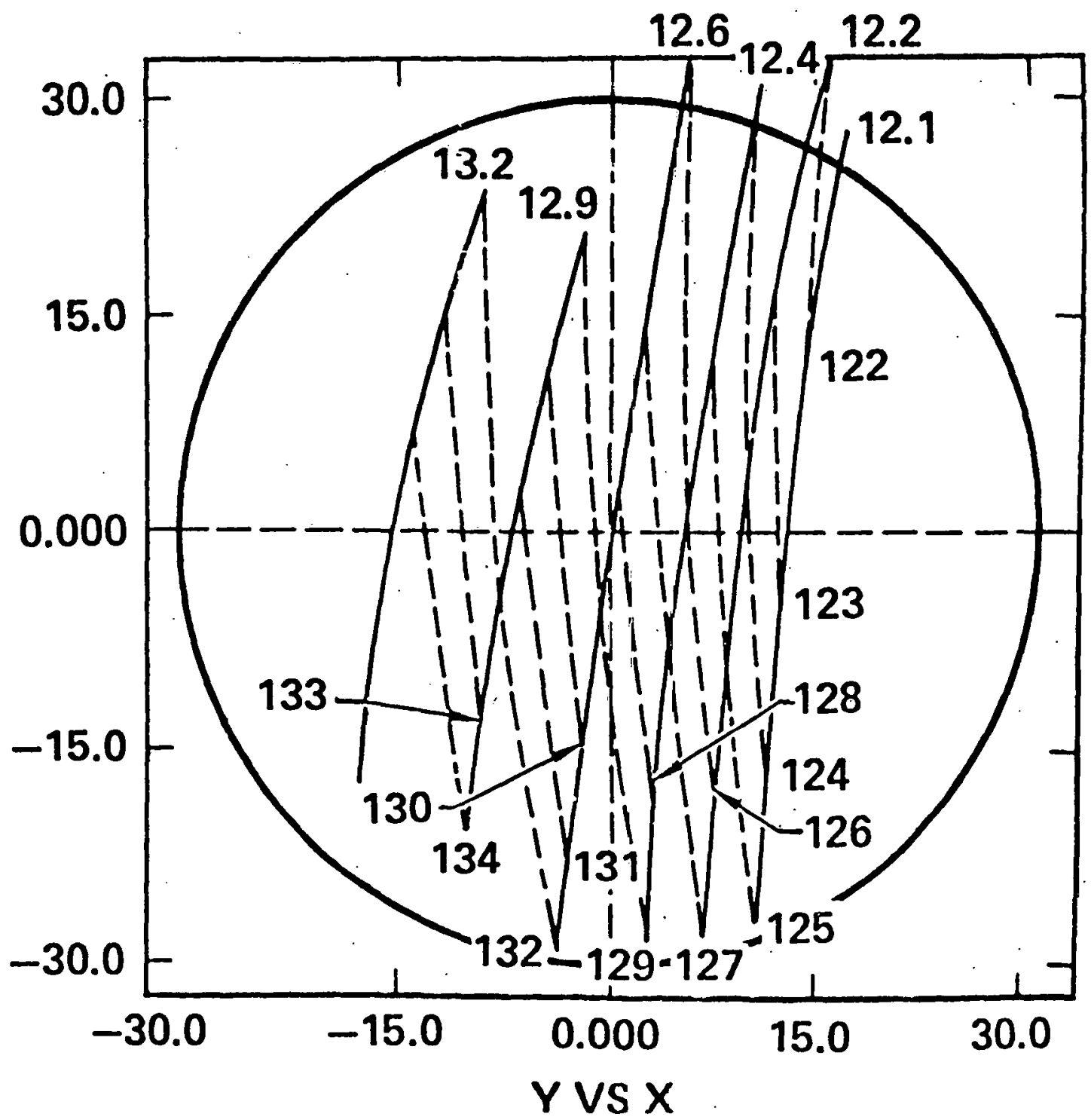

Fig. 11 TMX detection grif distorted by finite beta $(\beta=0.5)$ and space potential $(\theta=1000 \mathrm{~V})$ 
For real systems the magnetic field is almost always three dimensional. On tokamaks for example, if the beam remained exactly in the midplane between toroidal field coils, the toroidal field would only have a toroidal component. As soon as you move out of the midplane, there are radial components to this field. The poloidal field due to the plasma current pushes the beam out of the midplane and it then samples the non-toroidal components of the toroidal field. This complicates the analysis, but it is still possible to carry it out. In any case it is still necessary to take the derivative of experimental data, and this is always subject to considerable error. The displacement of the beam due to the poloidal field of a tokamak has been observed, but no measurements of the poloidal field structure has been carried out. It does not appear that this would be a very sensitive way of trying to evaluate the plasma current density distribution.

For strongly three dimensional magnetic fields such as those produced by "basebal1" geometry, there are additional problems. Here it is not a question of measuring the magnetic field since it is known, but simply trying to carry out $\phi$ and $n F\left(T_{e}\right)$ measurements. The strong field gradients of this geometry severely defocuses the beam. An injected circular beam will have an elongated elliptical cross section at the center of the field. Space resolution can be maintained by using an aperatured detector that only looks at a small fraction of the elliptical cross section, but this considerabiy reduces the secondary ion signal. In Sect. IX we discuss a variation that may avoid some of these problems. At the present time there have been no successful beam probe measurements in "baseball" geometry, but we expect to make some in the coming year on the ALEX facility at RPI. 


\section{PROBLEM AREAS}

The major problem, of course, is noise. When beam probe systems are brought on the air, the major initial noise is generally due to ground loops. We are looking for nanoamp signals on devices where there are kiloamp currents and it is very easy for some obscure ground loop to generate large noise currents in the detector. With enough hard work the ground loops can generally be eliminated and we get down to the basic noise level of the system.

On most systems the basic noise problem is due to ultra-violet light from the plasma entering the entrance aperature of the analyzer, and then scattering down to the detector plates where it generates photo-electrons. If the magnetic field radius is considerably larger than the plasma radius, then the beam will follow a curved path after it leaves the plasma and it is possible to orient the detector so that there is no line of sight back to the plasma. Usually we are not this fortunate. The problem can be reduced by using a screen for the top plate of the analyzer which transmits most of the UV coming in the entrance aperature.

On TMX we ran into a new source of noise. They have a 50 amp diagnostic neutral beam on the center cell. This beam is aimed such that it should not enter the analyzer and even if it did, it should not get transmitted to the detector plates. With a 50 amp beam, however, there is enough scattering that a few nanoamps ends up on our detector.

One way to improve the signal-to-noise ratio is to use a chopped beam and a phase sensitive or narrow band detector. This technique is in routine use on the EBT beam system where we use a $3 \mathrm{KHz}$ chop frequency, phase 
sensitive detection with very long integration time. The long integration time is possible because this is a dc device. With this technique, we can obtain a sensitivity of the order of 0.01 nanoamps. On TMX and RENTOR we are also using a chopped beam, but have not implemented narrow band detection. At the present time we are leaning towards simply recording the data and then using off line digital filtering. This should provide greater flexibility in the data reduction process.

Plasma access is another major problem on most installations. Mapping a major portion of the plasma and optimizing the detection grid requires large access ports and these are generally at a premium on most plasma experiments. If the magnetic field is larger than the plasma, then curvature of the beam can complicate the situation since most access ports are straight line. Ideally we would like to specify the access ports and have them specially fabricated for the beam probe system, but normally the access ports are already in place and we have to design around them. The exception was an EBT where ORNL fabricated a new cavity with the access ports we specified.

As noted earlier, beam attenuation can be a problem on large experiments. On present day systems, this is not a mjaor problem. Although attenuation is not negligible, it can be corrected for and it is still possible to deliver sufficient primary beam current all regions of the plasma. On larger systems, however, the attenuation could become so severe that it would be impossible to penetrate the full plasma diameter. More information on multiple ionization cross sections is needed. Hughes and Feeney (Ref. 28) have recently completed measurements on the 
$3^{+}$and $4^{+}$cross sections for $\mathrm{Rb}$, but we need information on $\mathrm{CS}$ and $\mathrm{Tl}$. It is also important to obtain cross-section data for higher electron energies as the temperature of plasma systems continue to increase. It is also possible that some of the $2^{+}$secondary ions are being created by charge exchange. There is practically no information on cross section for charge exchange reactions of the form $\mathrm{R}^{+}+\mathrm{H}^{+} \rightarrow \mathrm{R}^{++}+\mathrm{H}^{0}$.

Considerably more work needs to be done on improving ion guns, ion optics, energy analyzers, and ion sources. We have spent a fair amount of work on improving energy analyzers, and are reasonably satisfied with the present versions. We are, however, testing an analyzer with curved split plate detectors that should reduce the sensitive to misalignment in the $z$ direction. Electrostatic energy analyzers should be useful up to a few hundred KeV, but for higher energy beam systems some combination of magnetic and electrostatic analyzers look more attractive. Improvement in ion guns and ion optics should be capable of providing an order of magnitude increase in beam currents. This would certainly reduce the signal-to-noise problem.

\section{VARIATIONS}

The major variation of the type of beam probing discussed in this report is the use of neutral primary beam instead of the singly ionized primary. As you would expect this has its advantages and its disadvantages. The primary advantage is that it reduces the energy requirements to penetrate the same magnetic field and the primary beam will not be affected by strong magnetic field gradients. The disadvantages are the requirement for a neutralization cell on the primary beam line and the loss of flexibility in 
controlling and sweeping the primary beam. The 0 to $1^{+}$reaction also has a higher cross section for low electron temperatures, and this can be an advantage or a disadvantage depending on the particular system.

The beam energy required to penetrate a given magnetic field scales as $R^{2} B^{2}$ where $R$ is the radius of the field and $B$ the intensity. The major requirement is that the radius of curvature of the secondary ion is greater than $R$. The radius of curvature is proportional to $E^{1 / 2} / q$, so by reducing $q$ by a factor of 2 the energy requirement can be reduced by a factor of 4 . The straight line trajectory of the neutral primary can also be an advantage on some systems with limit access port structures.

The energy requirements for the primary beam may not be dictated by the magnetic field. It has been pointed out that the beam attenuation can be a problem particularly for large high density plasmas. Recall that the effective cross section is inversely proportional to the beam velocity; consequently, the attenuation decreases with increasing beam energy. On some systems this may be the determining factor in selecting the beam energy requirements.

It was noted earlier that for strongly 3 dimensional magnetic systems such as "basebaij" geometry, the field gradients severely defocus the primary beam. This can be alleviated by using a neutral primary. The field gradients still effect the $1^{+}$secondary ions, but at least half the problem has been eliminated. Simulation studies indicate that this technique should provide considerable increase in signal levels for the same space resolution. A neutral beam system of this type is presently under development on the ALEX "baseball" facility at RPI. 
To generate the neutral beam, an ion beam is accelerated to the proper energy and then passed through a neutralizing cell. For the alkali metal systems, this is not too difficult since the neutralizing cell can make use of the metal vapor and resonant charge exchange. By using cold baffles along the neutral beam line, it is relatively easy to limit the flux of cold metal vapor atoms from reaching the plasma.

Swecping or stecring of the neutral beam is a major problem. Il can be done mechanically, but this is slow and cumbersome. The only easy knob that you have control of is the beam energy. This will permit profile measurements along a predetermined line through the plasma, but it will not permit mapping of the plasma cross-section. A single profile measurement, however, can be of significant importance on a number of plasma devices.

Another variation that has been proposed for mirror systems is to send an ionized primary beam directly down the magnetic axis and then measure the energy of the secondary ions at the other end. This would provide a direct measurement of the space potential, but would not provide spatial resolution. This technique looks attractive for a device like TMX where it may be able to shed some light on the potential difference between the end plugs and center cell. It is exceedingly difficult, however, to direct. a beam precisely along the magnetic axis. If the magnetic axis is not a straight line or the injection beam makes a very small angle with respect to the axis, it will follow a curved path and most likely end up running into the wall. 
X. COST AND SCALING

Typical cost for a present day beam probe system operating in the 10 to $100 \mathrm{KeV}$ range is in the range of $\$ 200 \mathrm{~K}$ to $\$ 500 \mathrm{~K}$. Most of this cost is for salary and computer time. Actual hardware costs are probably about $1 / 4$ of the total cost. Since each system is specifically designed for a particular plasma device, there can be very large variations in cost depending on the sophistication desired and the equipment available in the laboratory. The largest variation is generally in the data acquisition area. Once a beam probe is in operation, it can produce copious quantities of data and the data handling and processing equipment can get quite elaborate. We have recently estimated the cost of a $500 \mathrm{KeV}$ system and our estimate is about $\$ 1000 \mathrm{~K}$. Roughly $\$ 250 \mathrm{~K}$ would be for equipment and the rest would be for man-power. Present day systems take roughly 1 year from start of design to initial operation. This could probably be reduced somewhat, but not by much since the major delay is in hardware fabrication and equipment delivery.

As noted earlier the required beam energy scales with $R^{2} B^{2}$. Sys.tems presently in operation on EBT, TMX, and RENTOR are in the 10-100 KeV range. We are now considering $500 \mathrm{KeV}$ beam probe systems which would be useful on ISX, TEXT, and EBT-P. (It should be noted that edge potential measurements on ISX and TEXT could be made with $100 \mathrm{KeV}$ systems.) Scaling to $500 \mathrm{KeV}$ appears to be straightforward. Accelerators are available and standard parallel plate electrostatic energy analyzers should still be satisfactory. In fact the first beam probe system on a major device was the $200 \mathrm{KeV}$ system 
on the ST Tokamak. This operated very reliably, but was limited to center line magnetic fields of less than $20 \mathrm{kG}$. Rough estimates of beam probe systems for a machine the size of PDX indicate that it would require a 2 MeV beam, and for reactor size systems the required beam energy would be in the $10 \mathrm{MeV}$ range. This is the beam energy required to map the complete plasma cross-section. If the measurements were restricted to the outer edge of the plasma, then the energy requirements would be greatly reduced. The use of a neutral primary beam would reduce the energy requirements for field penetration, but the higher energy beams may still be necessary to penetrate the plasma.

Accelerators in the $10 \mathrm{MeV}$ range are available, but they are large and expensive. New accelerator developments that would reduce both the size and cost would be extremely helpful. Conventional electrostatic energy analyzers are probably not useful above about $500 \mathrm{KeV}$, but combination of magnetic and electrostatic analyzers can be developed.

\section{TYPICAL RESULTS}

In this final section we will briefly present some typical measurements that have been made with beam probe systems. This is by no means an exhaustive list, but simply a small sample that illustrates the information that can be obtained with beam probes. The references listed at the end of the report will provide more detailed descriptions of beam probe systems and the measurements that have been obtained.

Figure 12 is a density profile taken on the ST Tokamak showing the radial localization of the Mirnov oscillations. The profile is cut off on 


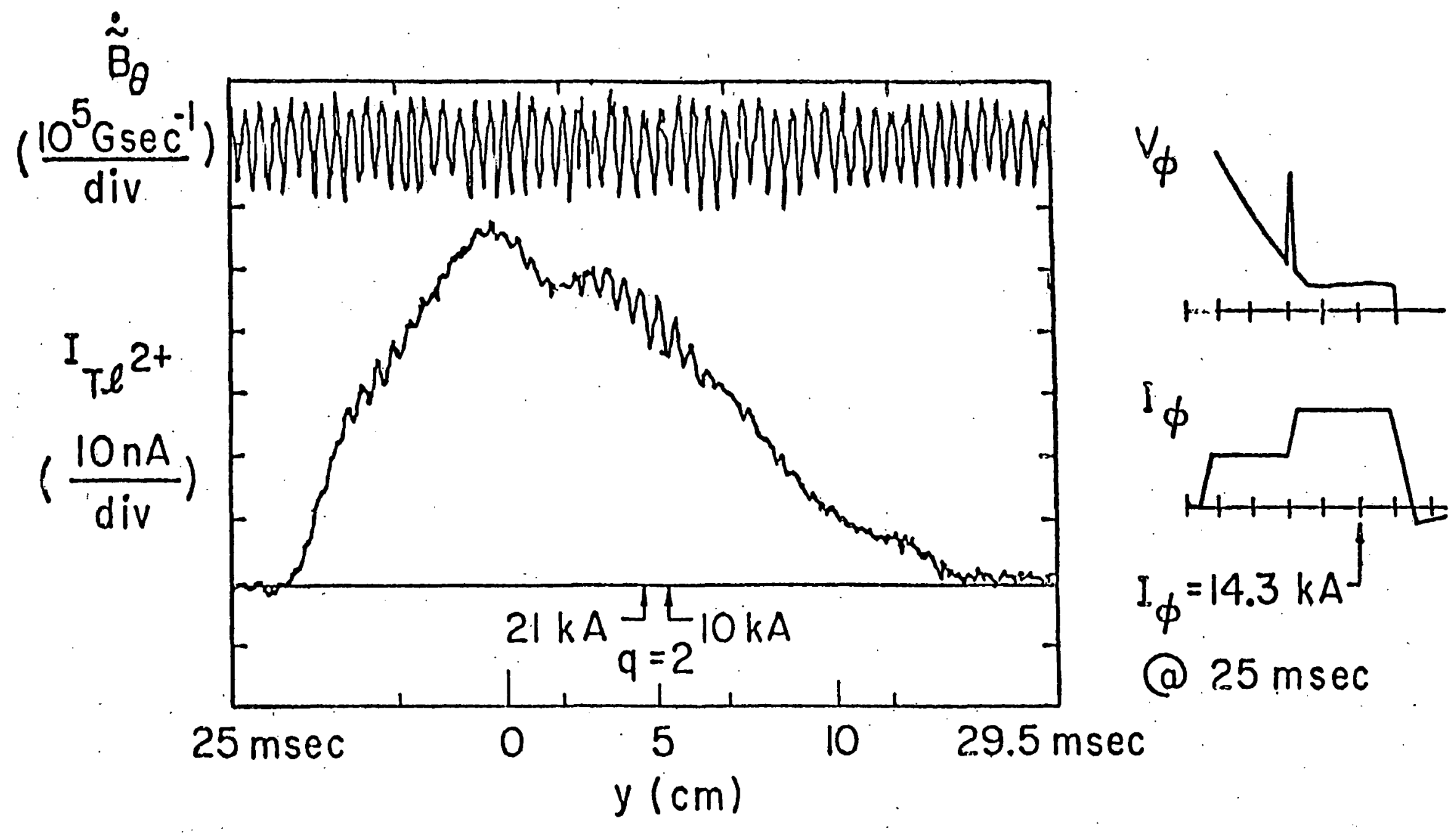

Fig. 12 Density Profile Measurements on the ST. Tokamak Showing the Localization of the Mirnov. Oscillations 
the left hand side due to the restricted size of the exit port. The upper trace shows the $B_{\theta}$ fluctuations picked up on magnetic loops located outside the plasma. This was the first measurement that showed the localization of MHD oscillations inside a plasma. Corresponding space potential measurements showed that this disturbance propagated at the electron diamagnetic velocity after correction for $E \times B$ rotation.

Figure 13 shows some density and temperature measurements on a hollow cathode arc at RPI. These measurements were made using $\mathrm{Na}^{+}$and $\mathrm{K}^{+}$beams .

A coherent fluctuation at approximately $70 \mathrm{KHz}$ was present in this hollow cathode arc, and Fig. 14 shows the density and potential fluctuations. These measurements were taken at a single point in the plasma and what is shown is a multitrace scope photograph. The D.C. level has been suppressed in both cases and narrow band filters were used to screen out random noise. The $\tilde{n}$ and $\tilde{\phi}$ measurements were made simultaneously and the phase difference between the two signals is easily observed. This is illustrated more clearly in the lower photograph which shows a Lissajous pattern. Phase measurements are very difficult to make and to insure that this was not an instrumental effect, the two amplifer channels were interchanged and the measurement repeated.

A contour map of the space potential in EBT is shown in Fig. 15. Note the symmetry near the center of the system. The symmetry begins to breakdown as you approach the last closed drift surface.

As noted earlier, we have observed $3^{+}$and $4^{+}$signals on EBT as well as the normal $2^{+}$signal. Figure 16 shows the intensity of the $3^{+}$signal relative to the $2^{+}$signal as you scan the observation point through the hot 

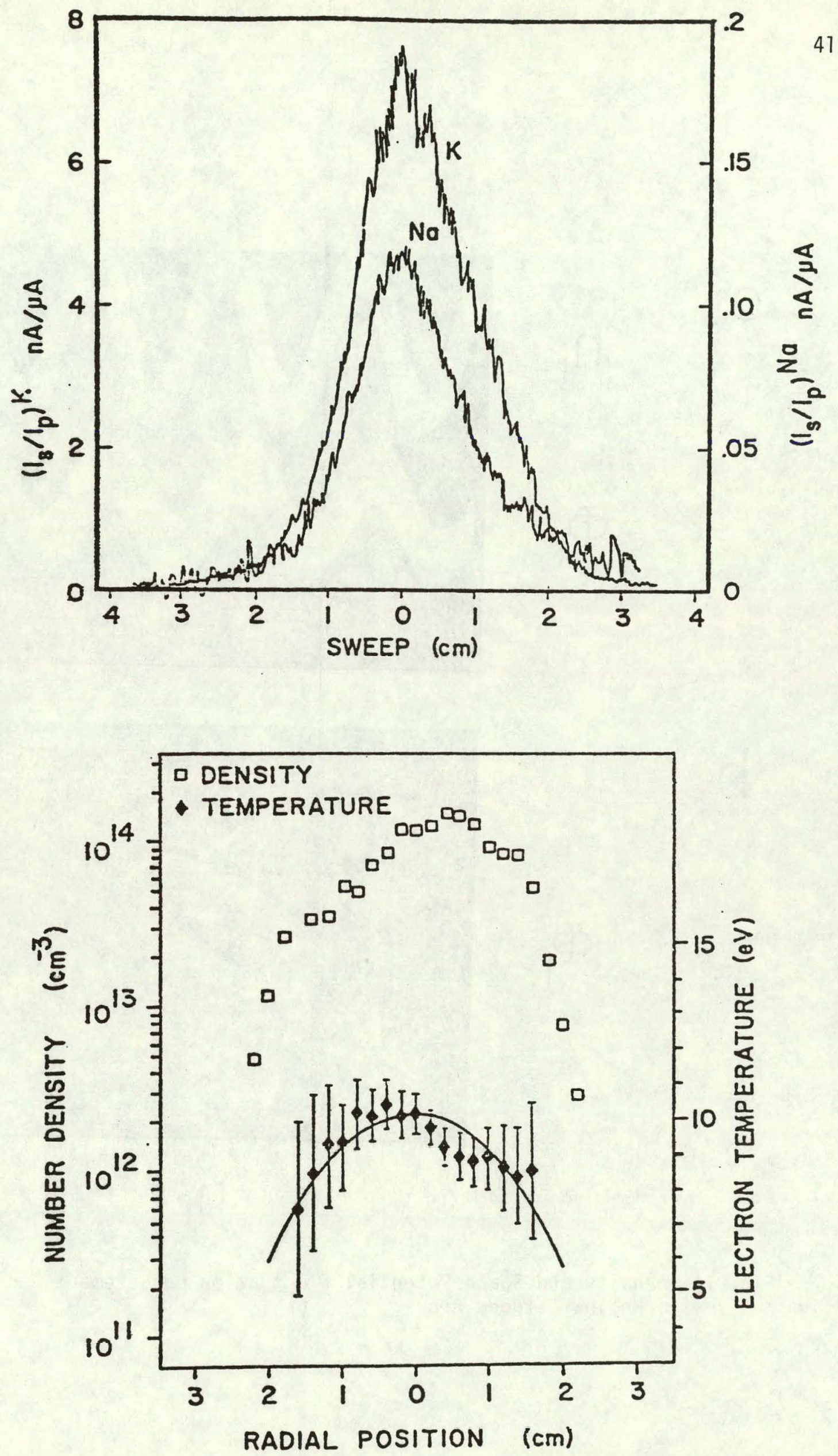

Fig. 13 Density and Temperature Measurements on a Hollow Cathode ArC 


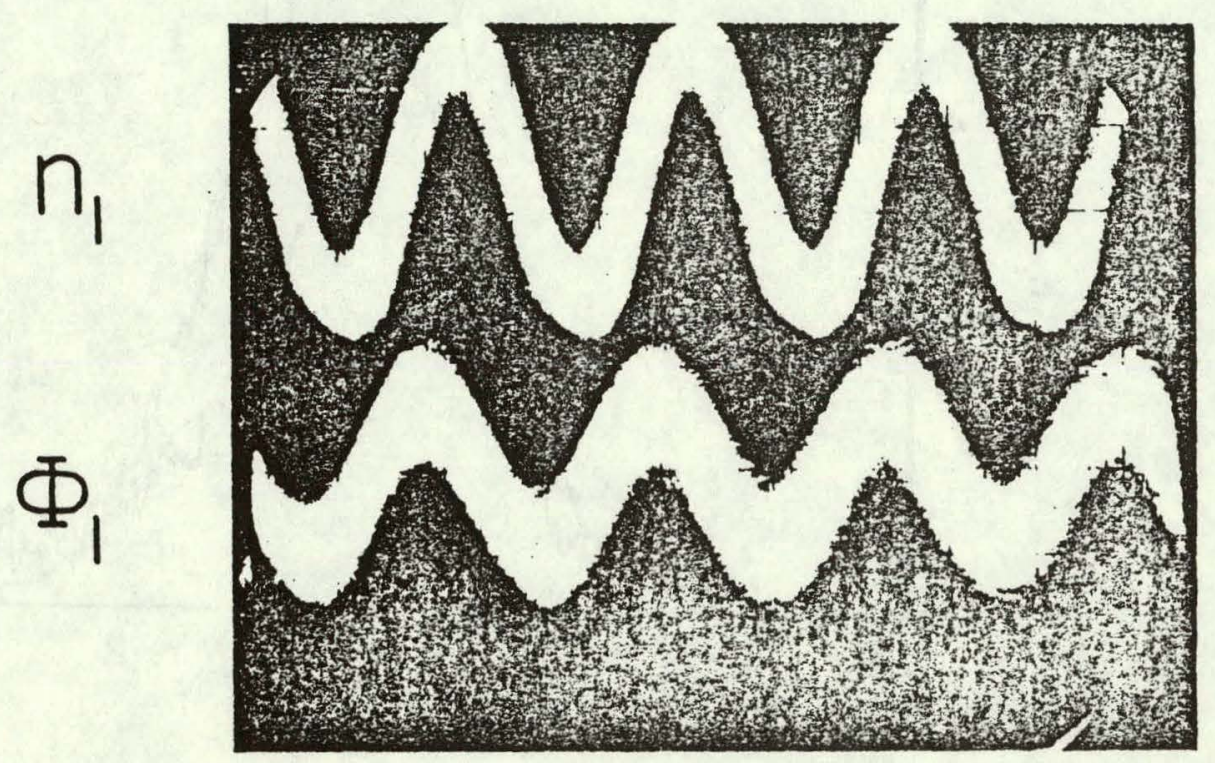

b

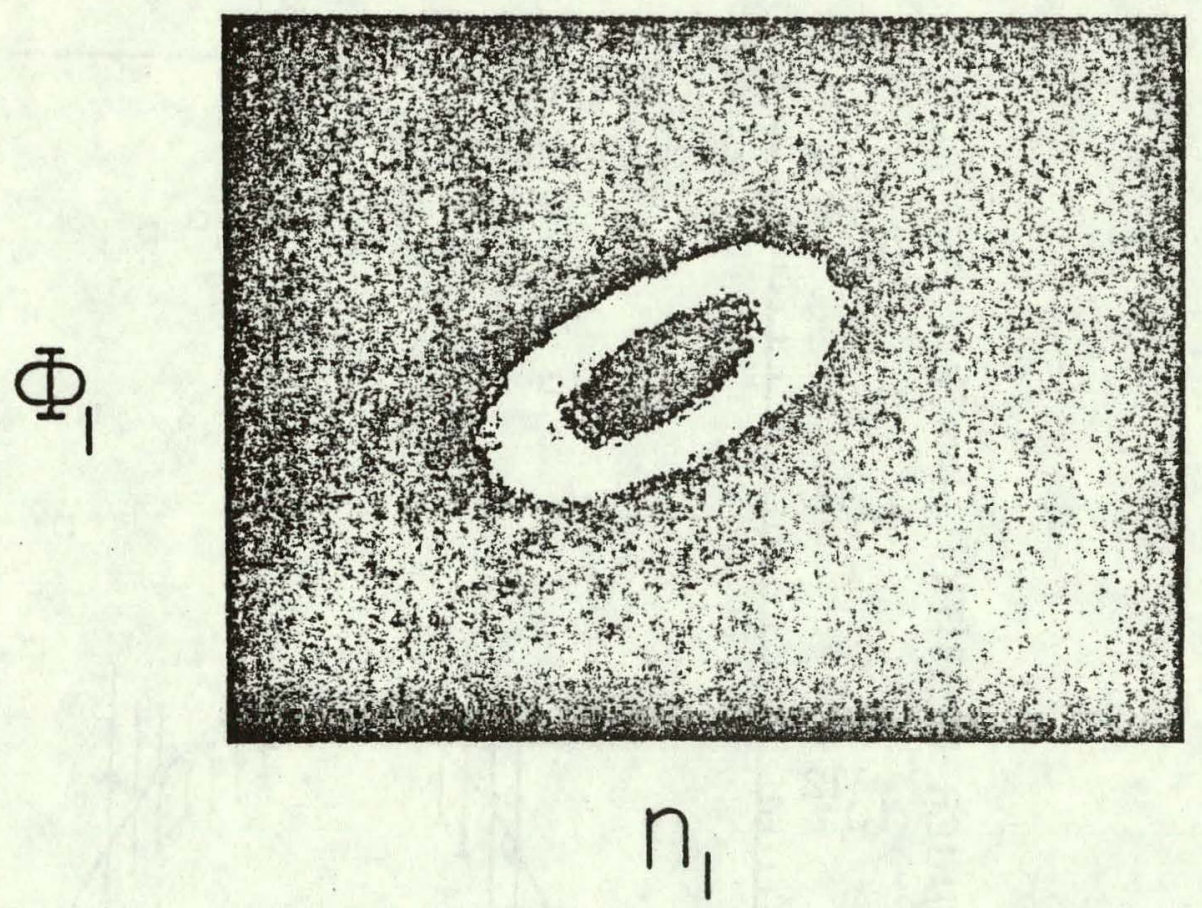

Fig. 14 Density and Space Potential Fluctuation Measurements on a Hollow Cathode Arc 


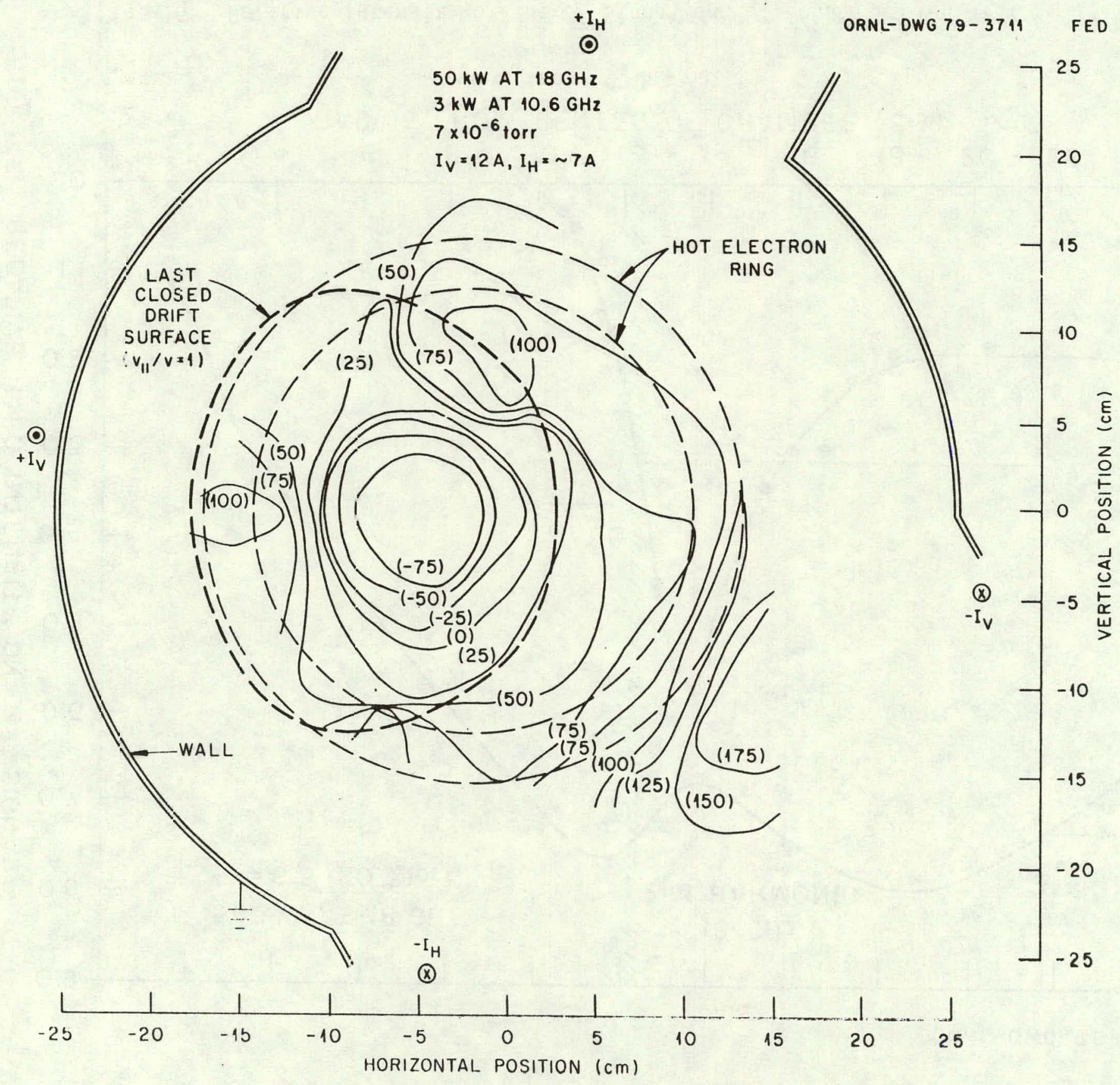

Fig. 15 Space Potential Contour Map on EBT 
ORNL-DWG 79-3459 FED

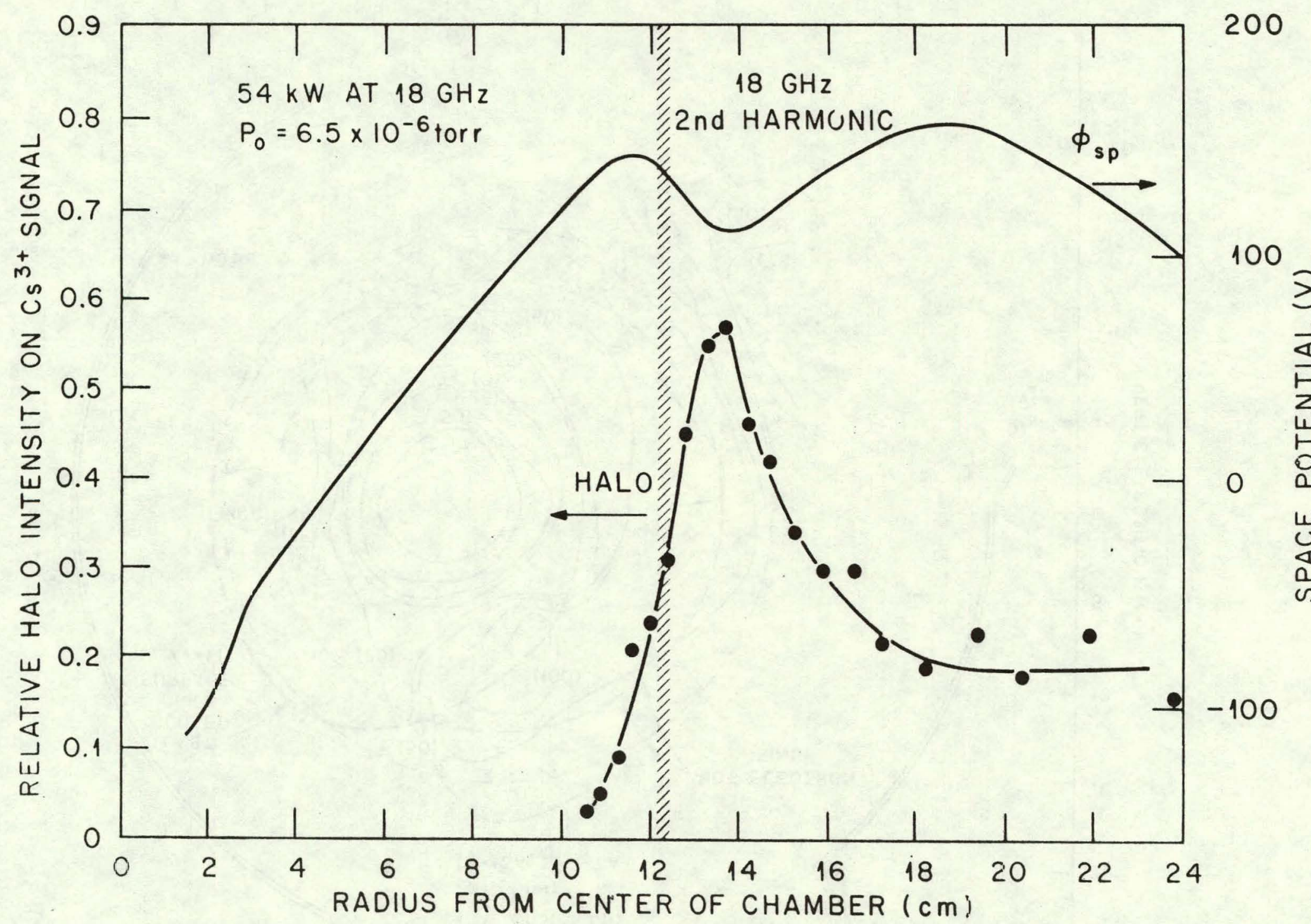

Fig. 16 Relative Intensity of the $3^{+}$Signal on EBT in the Region of the Hot Electron Ring 
electron ring region. This is referred to as the $3^{+}$halo signal. There is a rather abrupt change in the temperature of the background plasma as you pass through the ring region and if the curve is corrected for this change, then the peak in the halo falls on top of the $18 \mathrm{GHz}$ second harmonic heating zone.

Figure 17 shows some unprocessed secondary ion signals from the center cell of TMX. The beam is being swept across the analyzer and the peaks correspond to the secondary ion current. The small relatively flat D.C. offset is the noise level generated by the 50 amp diagnostic neutral hydrogen beam that was discussed earlier. The data was taken with a $12 \mathrm{KeV} \mathrm{Tl}^{+}$ion beam.

$3^{+}$secondary ions have also been observed on TMX and this data is shown in Fig. 18. The $3^{+}$signals are almost as large as the $2^{+}$signal, but note that this is raw data. To observe the $3^{+}$signal, the beam energy was increased to $20 \mathrm{KeV}$ and the primary beam current increases considerably as the energy goes up.

Finally some very preliminary data from the RENTOR Tokamak is shown in Fig. 19. Again the beam is being swept across the analyzer and the top trace in each photograph shows the sweep signal and the bottom two traces, the signals from the split plates. This is a $6 \mathrm{msec}$ pulse plasma and the top photograph covers the first 2 milliseconds, the center the middle two milliseconds, and the bottom photograph the final two milliseconds. Initially the plasma is very noisy and there is not much useful information in the top photograph. The beam signals are clearly observable during the 2 to 4 millisecond period. There is a large burst of noise as the plasma 


\section{Setup}

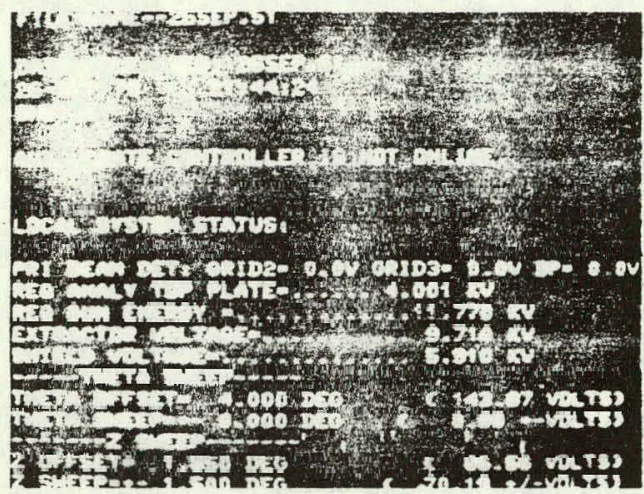

Front of analyzer

West
Split plate signels
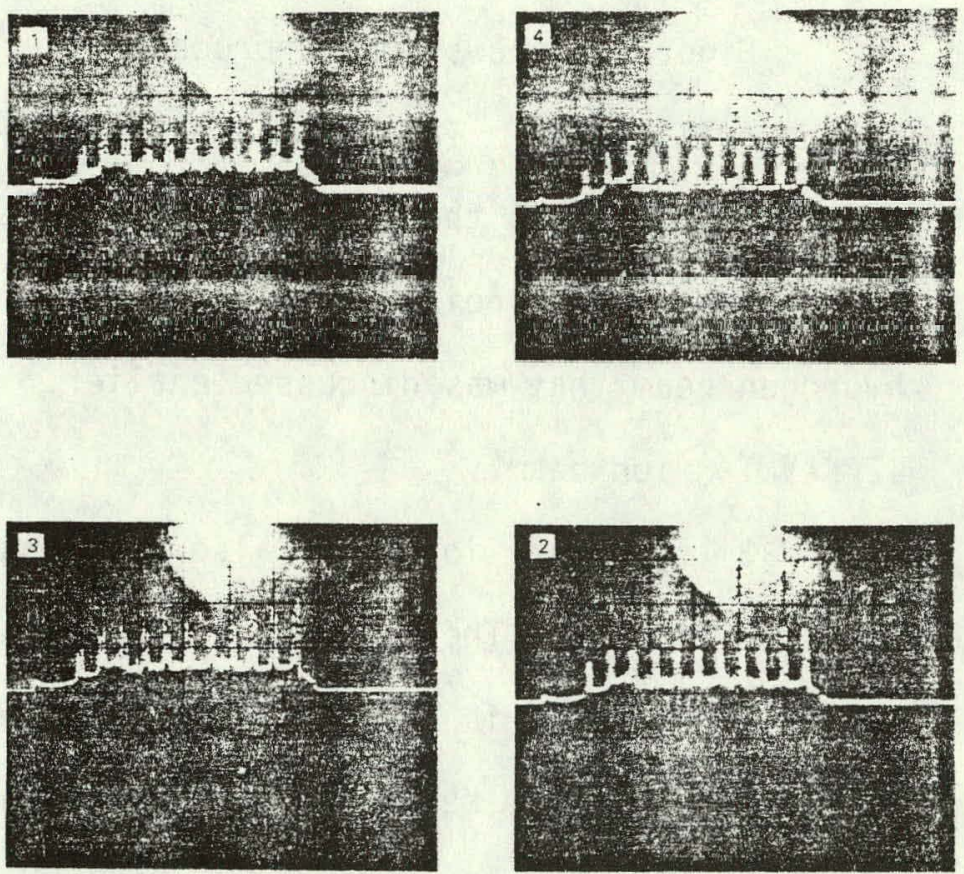

Horizontal: $4 \mathrm{~ms} / \mathrm{div}$

To separate the secondary signal from the background noise the beam is chopped eastVertical: $20 \mathrm{na} / \mathrm{div}$ west across the split plates. This is done by sweeping the primary beam with the " $z$ " plates. Note that in this shot the beam is swept too far west - out of the analyzer entrance slit. This gives the double peaked signals on plates 1 and 3. 


\section{Setup}

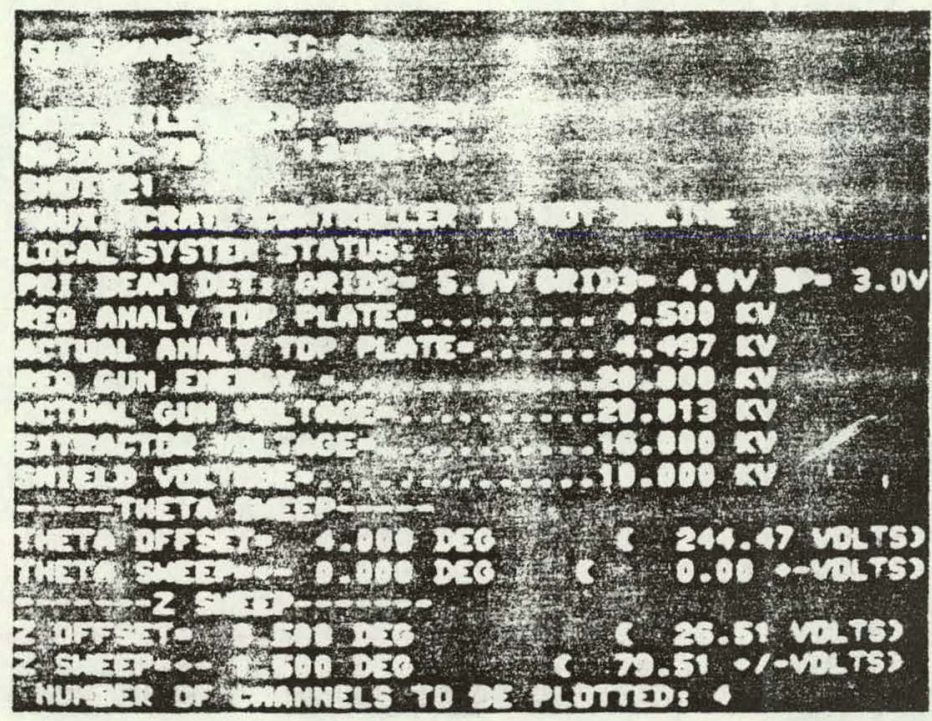

\section{Split plate signals}

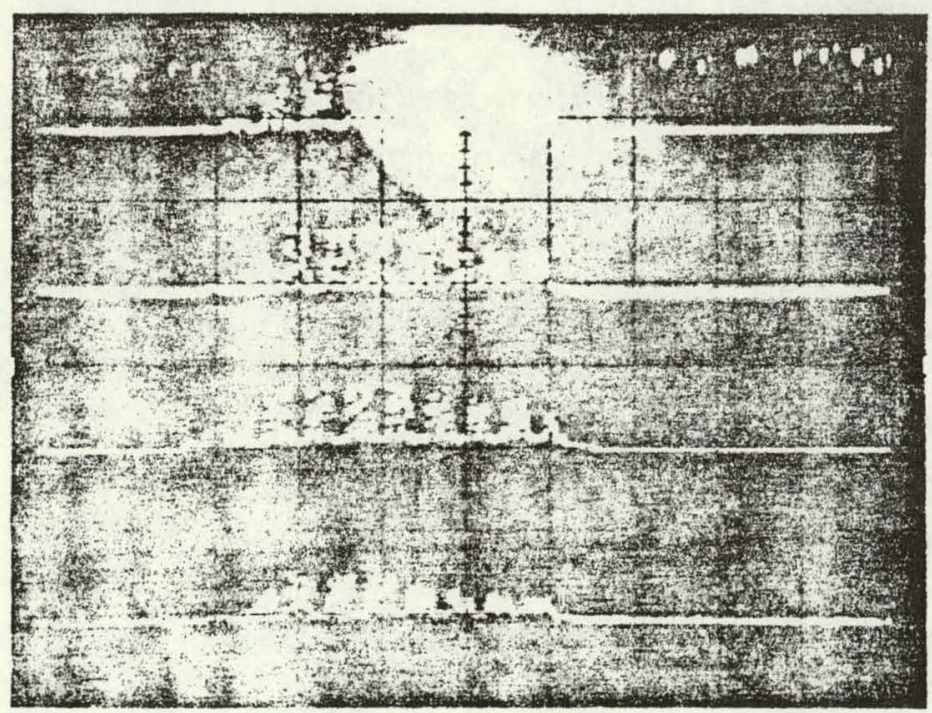

\section{Reaction}

$$
\mathrm{T}^{+}+\mathrm{e}^{-} \rightarrow \mathrm{T} \ell^{+++}+3 \mathrm{e}^{-}
$$

Tertiary current level

$$
I_{t}=3 I_{p} \ln \sigma_{e f f, t}\left(T_{e}\right)
$$

Tertiary data may be combined with secondary data from the same plasma volume to yieid a direct measurement of $T_{e}$ :

SP1

$$
\frac{I_{s}}{I_{t}}=\frac{2 I_{p} \ell_{s} n_{e} \sigma_{\text {eff, } s}\left(T_{e}\right)}{3 I_{p} \ell_{t} n_{e} \sigma_{\text {eff, } t}\left(T_{e}\right)}=\frac{2 \ell_{s} \sigma_{\text {eff, } s}\left(T_{e}\right)}{3 \ell_{t} \sigma_{\text {eff, } t}\left(T_{e}\right)}
$$

SP2

SP3

$5 P 4$

Horizontal: $4 \mathrm{~ms} / \mathrm{div}$

Vertical: $100 \mathrm{na} / \mathrm{div}$

Fig. $18 \mathrm{~T}^{3+}$ Signals from TMX 


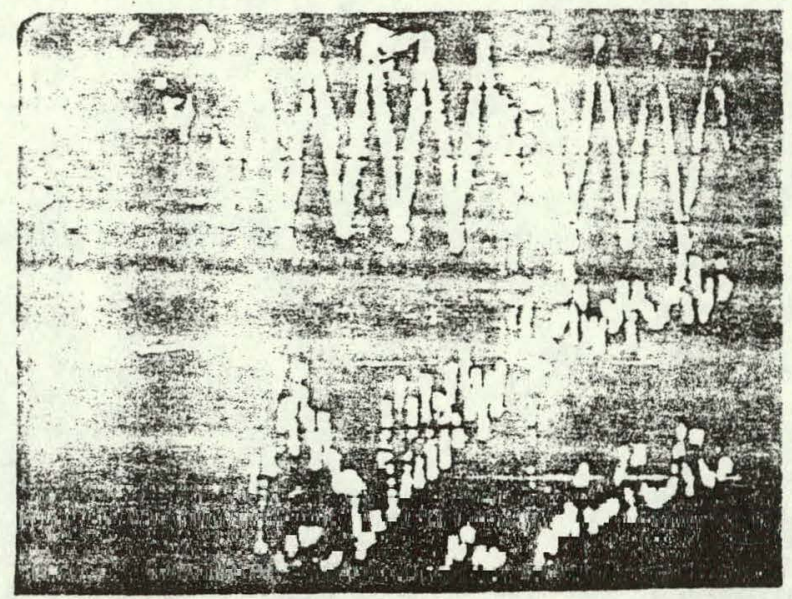

\author{
1/5/80 Shot \#108 \\ PANORAMIC VIEW OF THE \\ DETECTOR CHANNELS
}

RADIAL SWEEP VOLTAGE: ${ }_{\mathrm{X}} \mathrm{O} \mathrm{V} \mathrm{V} / \mathrm{CH}$ a $\mathrm{G} \mathrm{KHZ}$ $-482.5 \vee$ OFFSET

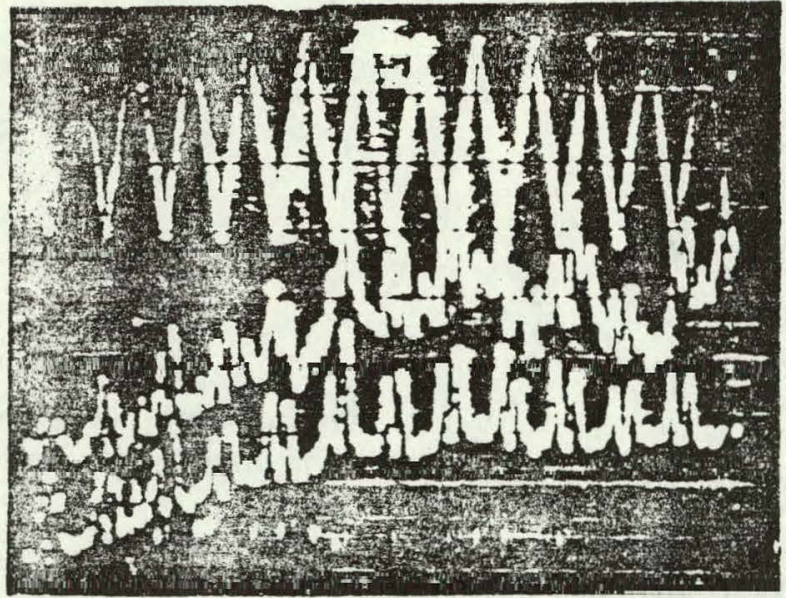

SECONDARY ION CURRENT:

FRONT SFLIT PLATE:

$10 \mathrm{NA} / \mathrm{CM}$

REAR SPLIT PLATE:

$10 \mathrm{NA} / \mathrm{CM}$

TIME: $0.2 \mathrm{~ms} / \mathrm{CM}$
$\mathrm{RB}^{+}$PRIMARY ION BEAM, $19,420 \mathrm{~V}$

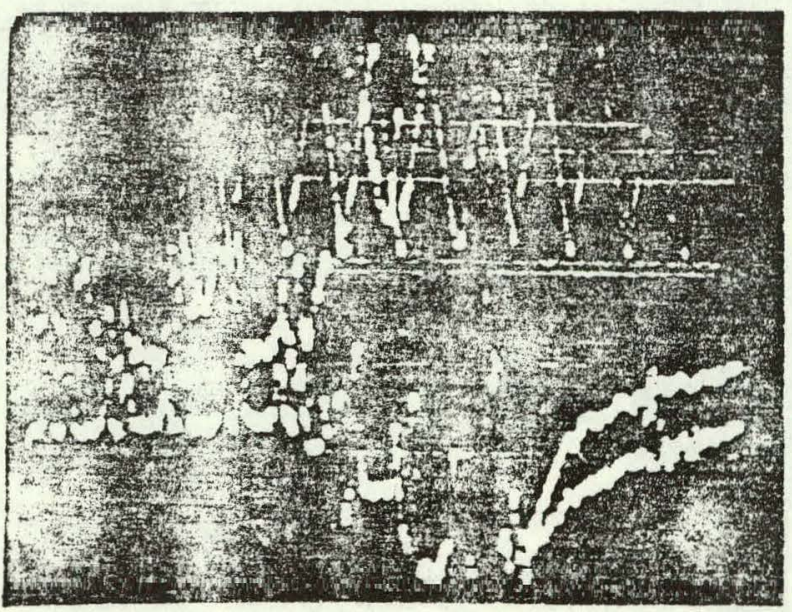

ANALYZER VOLTAGE $=4747 \mathrm{~V}$

Fig. 19 Beam Probe Signals from the RENTOR Tokamak 
begins to scrape off on the wall at about 5 milliseconds, but the beam probe signals aiso increase strongly during this period. We are just starting to analyze this data to find out what it is telling us about the plasma. 


\section{REFERENCES}

1. R. E. Reinovsky, Ph.D. Thesis, RPI (1973).

2. J. C. Glowienka, M.S. Thesis, RPI (1973).

3. J. C. Glowienka, Ph.D. Thesis, RPI (1975).

4. C. L. Fiore, M.S. Thesis, RPI (1978).

5. F. M. Bieniosek, M.S. Thesis, RPI (1977).

6. F. M. Bieniosek, Ph.D. Thesis, RPI (1980).

7. R. L. Hickok, "Plasmla Density Measurements by Molecular Ion Breakup", Rev. Sci. Inst., 38, 142 (1967).

8. F. C. Jobes and R. L. Hickok, "Plasma Density Contour Measurements Using Molecular Ion Beam Breakup", Rev. Sci. Inst., 38, 928 (1967).

9. F. C. Jobes, J. F. Marsha 11, and R. L. Hickok, "Plasma Density Measurement by Ion-Beam Probing", Phys. Rev. Letters, 22, 1042 (1969).

10. F: C. Jobes and R. L. Hickok, "A Direct Measurement of Plasma Space Potential", Nuc. Fusion, 10, 195 (1970).

11. J. C. Hosea, F. C. Jobes, R. L. Hickok, and A. N. Dellis, "Rotation and Structure of Low Frequency Oscillations Inside the ST Tokamak Plasma", Phys. Rev. Letters, 30, 839 (1973).

12. R. E. Reinovsky, W. C. Jennings, and R. L. Hickok, "Measurement of Plasma Electron Temperature with an Ion Beam Probe", Phys. Fluids, 16, 172 (1973).

13. R. E. Reinovksy, J. C. Glowienka, A. F. Seaver, and R. L. Hickok, "Ion Beam Probe Measurements of Electron Temperature", IEEE Trans. on Plasma Science, PS-2, 250 (1974).

14. W. C. Jennings and R. L. Hickok, "Space and Time Resolved Measurements in Magnetically Confined Plasmas Using a Heavy Ion Beam Probe", Proceedings of the 12th Int. Conf. on Phenomena in Ionized Gases 1975, North Holland Publishing Co., Amsterdam (1975).

15. W. C. Jennings, R. L. Hickok, and J. C. Glowienka, "Spatidlly Resulved Instability Measurements with a Heavy Ion Beam Probe", Proceedings of the 7 th European Conf. on Controlled Fusion and Plasma Physics, Ecole Polytechnique Federale, Lausanne, Switzerland (1975).

16. R. E. Reinovsky, J. C. Glowiena, W. C. Jennings, and R. L. Hickok, "Performance of a Feedback Controlled Electrostatic Energy Analyzer for Use with an Ion Beam Probe Diagnostic System", IEEE Trans. on Plasma Science, PS-3, 194 (1975). 
17. J. C. Glowienka, W. C. Jennings, and R. L. Hickok, "Spatially Resolved Measurements of Plasma Instabilities Using a Heavy Ion Beam Probe", Applied Physics Letters, 28, No. 9 (1976).

18. R. L. Hickok, F. M. Bieniosek, P. L. Colestock, K. A. Connor, and W. C. Jennings, "Space Potential Measurements on EBT", Proceedings of the 8th European Conf. on Controlled Fusion and Plasma Physics, Czechoslovakia, Academy of Sciences, Prague 8, Czechoslovakia (1977).

19. R. L. Hickok, W. C. Jennings, and K. A. Connor, "Heavy Ion Beam Probing", Proceedings of the II Conf. on Hot Plasma Diagnostics Atomizdat, Hoscow 103031, USSR (1977).

20. J. H. Stufflebeam, W. C. Jennings, and R. L. Hickok, "A Heavy Ion Beam Probe Plasma Diagnostic System for the Deep Magnetic Well LITE Device", IEEE Trans. Plasma Science, PS-6, 130 (1978).

21. P. L. Colestock, K. A. Connor, R. L. Hickok, and R. A. Dandl, "Direct Measurement of Plasma Space Potential on EBT", Phys. Rev. Letters, $\underline{40}$, No. 26 (June 26, 1978).

22: R. A. Dandle, et.al., "Experimental Study of Plasma Parameters in Elmo Bumpy Torus (EBT)", submitted to IAEA Mtg., Innsbruch, Austria (August 23-30, 1978).

23. R. A. Dandl, et.al., "Experimental and Theoretical Studies of the Elmo Bumpy Torus (EBT)", Proceedings of the Sixty IAEA Conf. on Plasma Physics and Controlled Nuclear Fusion Research, Innsbruch, Austria (August 1978).

24. R. L. Hickok, W. C. Jennings, K. A. Connor, and J. T. Woo, "Space Potential Measurements with Particle Beam Probes", Proceedings of the 8th European Conf. on Plasma Physics and Controlled Thermonuclear Fusion (September 1979).

25. F. M. Bieniosek, P. L. Colestock, K. A. Connor, R. L. Hickok, S. P. Kuo, and R. A. Dandl, "Elmo Bumpy Torus Heavy Ion Probe", Rev. Sci. Inst., 52, $206(1980)$.

26. G. Tonetti and K. A. Connor, "The Direct Sensitivity of Particle Probes to Magnetic Fields of Hot Dense Plasmas", Plasma Physics (accepted for publication.

27. R. L. Hickok and F. C. Jobes, "Ion Beam Probe for the ST Tokamak", AFOSR Report TR-72-0018 (1971).

28. D. W. Hughes and R. K. Feeney, "Absolute Experimental Cross Sections for the Electron Impact Ionization of Rubidium", Georgia Inst. of Technology Report No. ORO-3027-52. 
Internai
K. $\therefore$ Connor
R. :. Hickok
W. C. Jennings
J. F. Lewis
R. .. Rafun
J. i. $\$ 00$
R.P.E.L. Library
R.P.C.L. Graduate itudents

\section{External}
P. Stone -- DOE
P. Colestock -- PPPL
R. Tàjlor -- UiL.:
H. Fleishman -- Cornell
J. C. Sprott -. ¿. $0=$ hisconsin
K. Moses -- TRW
J. .. Shohet -. J. $0=$ uis cons in
J. R. Roth -- U. of Tennessee
M. Kristiansen -- exas Tech
P. Willis - - GE
K. Gentile - - J. $c^{-}$-exas
D. Priester -- DOE
E. Pcivers -. U. if $T \equiv x \equiv S$
C. Damim -- LLL
J. L. Cecchi -. $D F=L$
P. Demchenko -- Kharkov
F. C Jobes -- JF=L
I.. Krupnik -. Kharkov
j. C. Glowienka -- O.ZN-
V. Tershin -- Kharkov
3. F. Pipkins -- $E \equiv n \equiv r \equiv$ ? Atomic
V. Yakubouski -- Kharkov
K. :icCormick -- jerching
J. Fljita -- Nac J' Jniversity
F. Vendenplas -- E:ole Royale Militaire
F. Cáp -- U. of Ir.usjuck
R. K. Feeney -- SEJrgia Tech
f. Dellis -- Culthe.s láb
h. Ard -. McDonreil Deuglas 